\title{
Tumour-Associated Macrophage Polarisation Promotes Progression of Esophageal Carcinoma
}

\section{Xin Yuan}

Shihezi University School of Medicine https://orcid.org/0000-0002-6375-3259

\section{Ya Li}

Department of Pathology and Key Laboratory for Xinjiang Endemic and Ethnic Diseases (Ministry of Education) / Department of Pathology, the First Affiliated Hospital, Shihezi University School of Medicine, Xinjiang 832002, China

\section{An Zhi Zhang}

Department of Pathology and Key Laboratory for Xinjiang Endemic and Ethnic Diseases (Ministry of Education) / Department of Pathology, the First Affiliated Hospital, Shihezi University School of Medicine, Xinjiang 832002, China

\section{Chen Hao Jiang}

Department of Pathology and Key Laboratory for Xinjiang Endemic and Ethnic Diseases (Ministry of Education) / Department of Pathology, the First Affiliated Hospital, Shihezi University School of Medicine, Xinjiang 832002, China

\section{Fan Ping Li}

Department of Pathology and Key Laboratory for Xinjiang Endemic and Ethnic Diseases (Ministry of Education) / Department of Pathology, the First Affiliated Hospital, Shihezi University School of Medicine, Xinjiang 832002, China

\section{Yu Fang Xie}

Department of Pathology and Key Laboratory for Xinjiang Endemic and Ethnic Diseases (Ministry of Education) / Department of Pathology, the First Affiliated Hospital, Shihezi University School of Medicine, Xinjiang 832002, China

\section{Jiang Fen Li}

Department of Pathology and Key Laboratory for Xinjiang Endemic and Ethnic Diseases (Ministry of Education) / Department of Pathology, the First Affiliated Hospital, Shihezi University School of Medicine, Xinjiang 832002, China

\section{Wei Hua Liang}

Department of Pathology and Key Laboratory for Xinjiang Endemic and Ethnic Diseases (Ministry of Education) / Department of Pathology, the First Affiliated Hospital, Shihezi University School of Medicine, Xinjiang 832002, China

\section{Hai Jun Zhang}

Department of Pathology and Key Laboratory for Xinjiang Endemic and Ethnic Diseases (Ministry of Education) / Department of Pathology, the First Affiliated Hospital, Shihezi University School of Medicine, 
Xinjiang 832002, China

\section{Chun Xia Liu}

Department of Pathology and Key Laboratory for Xinjiang Endemic and Ethnic Diseases (Ministry of Education) / Department of Pathology, the First Affiliated Hospital, Shihezi University School of Medicine, Xinjiang 832002, China

\section{Li Juan Pang}

Department of Pathology and Key Laboratory for Xinjiang Endemic and Ethnic Diseases (Ministry of Education) / Department of Pathology, the First Affiliated Hospital, Shihezi University School of Medicine, Xinjiang 832002, China

\section{Xi Hua Shen}

Department of Pathology and Key Laboratory for Xinjiang Endemic and Ethnic Diseases (Ministry of Education) / Department of Pathology, the First Affiliated Hospital, Shihezi University School of Medicine, Xinjiang 832002, China

\section{Feng Li}

Department of Pathology and Key Laboratory for Xinjiang Endemic and Ethnic Diseases (Ministry of Education) / Department of Pathology, the First Affiliated Hospital, Shihezi University School of Medicine, Xinjiang 832002, China. Department of Pathology, Be

\section{Jian Ming Hu ( $\sim$ jianming.120@163.com )}

Department of Pathology and Key Laboratory for Xinjiang Endemic and Ethnic Diseases (Ministry of Education) / Department of Pathology, the First Affiliated Hospital, Shihezi University School of Medicine, Xinjiang 832002, China

\section{Research}

Keywords: Tumour-associated macrophage, FGL2, esophageal carcinoma, bioinformatics, tumourinfiltrating, functional network analysis

Posted Date: July 6th, 2020

DOl: https://doi.org/10.21203/rs.3.rs-38333/v1

License: (c) (i) This work is licensed under a Creative Commons Attribution 4.0 International License. Read Full License 


\section{Abstract}

Background: The immune response mediated by tumour-associated macrophages (TAMs) is vital in tumour progression in many cancers. Fibrinogen-like protein 2 (FGL2) is a critical immunosuppressive factor that regulates the tumour microenvironment. However, no study has yet reported on the relationship between FGL2, tumour-infiltrating lymphocyte recruitment, and prognosis in esophageal carcinoma (ESCA).

Methods: Differentially expressed genes (DEGs) in various macrophage phenotypes were analysed using the GEO database. We identified the hub genes involved in affecting ESCA clinical prognosis using Kaplan-Meier plotter. Correlations between hub genes and immune infiltrates were analysed using the Tumour Immune Estimation Resource (TIMER) database, while correlation analysis between FGL2 and cytokine expression was assessed using cBioPortal. In vitro cell co-culture experiments were performed to examine the role of FGL2 in promoting tumorigenesis. Finally, we compared the GO terms and KEGG pathways enriched by the DEGs in M1 and M2 macrophages using DAVID.

Results: High FGL2 expression was significantly associated with poorer overall survival and relapse-free survival of esophageal cancer patients. FGL2 expression was positively correlated with immune markers and infiltrating levels of $B$ cells, CD8+ T cells, CD4+ T cells, macrophages, neutrophils and dendritic cells. In addition, FGL2 expression was strongly correlated to IL-10, MMP9, CCL5, TIM-3, IL-13, VCAM1, M-CSF and FGF-7 expression.

Conclusions: M2-like TAMs may regulate the tumour microenvironment by secreting FGL2, thereby inducing the occurrence and progression of ESCA. Reversing TAM polarization may be an effective strategy that reveals new targets for immunotherapy in treating ESCA.

\section{Background}

Esophageal carcinoma (ESCA) is the sixth leading cause of cancer-related death and one of the eight most common malignancies [1]. Moreover, the incidence rate of esophageal squamous cell carcinoma (ESCC) is increasing globally. Although the diagnosis and treatment of ESCA have gradually improved, according to the latest statistical analysis, the five-year survival rate of ESCA is still around $10-20 \%$ [2]. This is mainly due to late diagnosis, metastatic tendency, and immune resistance. Immune escape and immunosuppression of tumour cells are important factors leading to tumour progression and poor prognosis of patients with ESCA.

Tumour growth is a chronic process that occurs gradually. The interaction between tumour cells and surrounding cells forms a complex and variable tumour microenvironment (TME), which determines the rate of progress and outcome of the tumour. To explore the distribution of tumour-infiltrating immune cells (TIICs) in the esophageal cancer microenvironment, we analysed ESCA samples in the TCGA database. As a result, the TIICs are mainly macrophages and T cells with different phenotypes, and their number accounts for $70 \%-80 \%$ in the esophageal cancer microenvironment (Fig. 1). The functional state 
of immune cells in the TME is one of the key mechanisms affecting tumour reversion. Its immune-related mechanisms play an important role in ESCA, and immunotherapy is considered a promising treatment of ESCA [3]. Therefore, there is an urgent need to understand the immunophenotypes involved in tumourimmunity interactions and identify novel immune-related therapeutic targets in esophageal carcinoma.

The multiple effects of tumour-associated macrophages (TAMs) on tumour cells in the TME are the focus of many current research studies. TAMs have a high degree of plasticity, and can be polarised by different stimuli into classic activated proinflammatory macrophages (M1-like TAMs) and alternative activated immunosuppressive macrophages (M2-like TAMs). The polarised M1 and M2 macrophages reflect the two extremes of TAM function, with M2 macrophages having a close association with the occurrence, progression and poor prognosis of cancer $[4,5]$. In addition, our previous studies showed that M2 macrophages in the TME promote the occurrence, invasion and migration of esophageal carcinoma cells and are closely related to poor patient prognosis $[6,7]$. Notably, the presence of cytokines in the TME allows TAMs to obtain a tumour-promoting phenotype (M2 macrophages), thereby exerting an immunosuppressive effect [8]. However, TAMs are essentially plastic cells that can be reprogrammed phenotypically to elicit an anti-tumour response. In other words, TAMs can be reprogrammed into the antitumour M1 macrophages [9].

Fibrinogen-like protein 2 (FGL2) is a member of the fibrinogen superfamily with prothrombin activity and multiple functions in immune regulation. FGL2 is highly expressed in various tumour tissues and plays a vital role in tumour occurrence and progression $[10,11,12]$. As an immunoregulatory factor, FGL2 has been shown to play a multimodal role in the innate and acquired immune responses, including enhanced activation of T regulatory cells (Tregs) and maintenance of their immunosuppressive activity, a balanced ratio of Th1 and Th2 cells, and inhibition of antigen-presenting activity $[13,14]$. Previous studies have shown that FGL2 inhibits the proliferation of effector T cells and the maturation of dendritic cells $[15,16]$. In addition, it promotes macrophage activation and induces B cell apoptosis [17]. Zhang, et al. demonstrated that FGL2 silencing leads to a significant decrease in the viability of renal cancer cells and increases cancer cell apoptosis, and high FGL2 expression is an independent factor leading to poor prognosis in patients with renal carcinoma [18]. In the TME, FGL2 is able to promote the transformation of M1 macrophages into tumour-promoting M2 macrophages by inducing CD39 expression [19]. However, the potential function and mechanism of FGL2 in cancer progression and tumour immunology of ESCA have yet to be reported.

In this study, we analysed the correlation between FGL2 expression and the prognosis of patients with ESCA in the Kaplan-Meier plotter database. We used the TIMER database to study the correlation between FGL2 and TIICs in the esophageal cancer microenvironment. Utilization the cBioPortal database and in vitro co-culture of different phenotypes of macrophages and esophageal cancer cells was performed to further study the correlation between FGL2 expression and cytokines, enabling us to explore the potential function and mechanism of FGL2 in ESCA progression and immunotherapy. Finally, enrichment of GO functions and KEGG pathways of differentially expressed genes (DEGs) in M1 and M2 macrophage 
samples with DAVID revealed strategies for reversing the polarization of tumour-associated macrophages, as well as new potential immunotherapies for treating esophageal carcinoma.

\section{Materials And Methods}

\subsection{Evaluation of TIICs in ESCA}

In this study, we used expression data from TCGA ESCA cohorts. The CIBERSORT [20] was performed to estimate the proportions of 22 TIICs both in ESCA samples and normal samples. To assess the accuracy of the deconvolution results, CIBERSORT derives a $\mathrm{p}$ value for each sample. Cases with CIBERSORT $\mathrm{P}<$ 0.05 was considered statistically significant difference. A total of 78 samples ( 2 normal samples and 76 ESCA samples in TCGA dataset) were filtered out. CIBERSORT software package was obtained from the developers and combined with R (R.3.6.1). We used the default signature matrix at ESCA permutations to analysis.

\subsection{Microarray data}

Genes were screened using the GEO (http://www.ncbi.nlm.nih.gov/geo) database, specifically the GSE57614 series [21] and the GSE36537 series [22] on the GPL6480 platform (Agilent-014850 Whole Human Genome Microarray $4 \times 44 \mathrm{~K}$ G4112F), the GSE5099 series [23-24] on the GPL96 platform (Affymetrix Human Genome U133A Array) and GPL97 platform (Affymetrix Human Genome U133B Array), and the GSE95405 series on the GPL570 platform (Affymetrix Human Genome U133 Plus 2.0 Array). According to the annotation information in each platform, probes were converted into corresponding gene symbols. The GSE57614 dataset contained 9 samples each of macrophage, M1 macrophage and M2 macrophage, respectively. The GSE36537 contained 9 samples each of macrophage, M1 macrophage and M2 macrophage, respectively. The GSE5099 contained 6 samples each of macrophage, M1 macrophage and M2 macrophage, respectively. The GSE95405 contained 3 samples each of M1 macrophage and M2 macrophage, respectively.

\subsection{Identification of DEGs}

GEO2R (https://www.ncbi.nlm.nih.gov/geo/geo2r/) was used to identify DEGs between macrophage and M2 macrophage samples, M1 macrophage and M2 macrophage samples, respectively. The parameters of macrophage and M2 macrophage samples were "adjusted P-value $<0.05,|\log F C| \geq 1.0$ ". The parameters of M1 macrophage and M2 macrophage samples were "adjusted $P$-value $<0.01,|\log F C| \geq$ 1.0". The Venn online tool (http://bioin forma tics.psb.ugent.be/webtools/Venn/) was carried out to identify significant DEGs, and overlapping DEGs of the Venn map were retained for further analysis.

\subsection{Selection and analysis of hub genes in macrophage and M2 macrophage samples}

Hub genes expression in ESCA samples was analyzed from TCGA using cBioPortal (https://www.cbioportal.org/) [25]. The correlation among hub genes expression, overall survival (OS), 
and recurrence-free survival (RFS) of patients with Esophageal Squamous Cell Carcinoma (ESCC) were analyzed using the Kaplan-Meier Plotter (http://kmplot.com/analysis/) [26], together with the hazard ratio (HR) and log-rank P-value. TIMER database is a comprehensive resource for systematic analysis of immune infiltrates across diverse cancer types (https://cistrome.shinyapps.io/timer/) [27], which infer the abundance of tumor-infiltrating immune cells (TIICs) from gene expression profiles according to the statistical method of a deconvolution previously published [28]. We analyzed the correlation of hub genes expression with the abundance of immune infiltrates, including CD $4+T$ cells, $C D 8+T$ cells, macrophages, $B$ cells, dendritic cells, and neutrophils, through gene modules. In addition, the hub gene expression levels against ESCA purity is displayed on the left-most panel [29].

\subsection{The correlation analysis between FGL2 expression and tumor-infiltrating immune cells}

We used correlation modules of TIMER to explore the correlations between FGL2 expression and gene markers of TIICs (monocytes, TAMs, M1macrophages, M2 macrophages, T cells (general), CD8 + T cells, B cells, Tregs, T-helper 1 (Th1) cells, T-helper 2 (Th2) cells, exhausted T cells, and etc.). These gene markers have been referenced in previous studies [30-32]. Moreover, the correlation module generates a scatter plot of expression between a pair of user-defined genes in ESCA, as well as Spearman's correlation and statistical significance. Gene expression level was shown with log2 RSEM. The online database Gene Expression Profiling Interactive Analysis (GEPIA) (http://gepia.cancer-pku.cn/index.html) [33] was used to further confirm the correlation between FGL2 expression and macrophages of different phenotypes. The ESCA and normal tissue datasets were used for analysis and the Spearman method was used to determine the correlation coefficient.

\subsection{The correlation analysis of FGL2 expression with coexpressed genes and cytokines and its GSEA analysis}

The LinkedOmics database (http://www.linkedomics.org/login.php) [34] was used to study genes differentially expressed in correlation with FGL2 in the TCGA ESCA cohort $(n=184)$. The Pearson method was used to determine the correlation coefficient.

The LinkFinder also generates statistical expression plots for individual genes, including volcano plots and heatmaps. Correlations between FGL2 expression and cytokines was analyzed which came from TCGA using cBioPortal. FGL2 gene set enrichment analysis (GSEA) was used to identify the functions or pathway activity variations involved in ESCA between low and high FGL2 gene mRNA expression phenotype. GSEA is a method used to determine whether a predefined gene set can show significant consistent differences in two biological states [35]. We used the GSEA software (version: 4.0.0) to study the potential effects of FGL2 expression on ESCA. Differences were screened using the normalized $p$ value $<0.05$ and FDR $<0.05$ criteria and 1000 gene set permutations were used in each analysis.

\subsection{Macrophage and ESCC cell co-culture}


The human cell line ESCC-EC109/9706 and leukemia THP-1 monocytes were obtained from the Fuxiang Biological Company (Shanghai, China). All cells were maintained in Roswell Park Memorial Institute (RPMI) 1640 medium (Gibco, Carlsbad, CA) supplemented with 10\% fetal bovine serum (FBS; Gibco) and cultured in a $5 \% \mathrm{CO} 2$ humidified incubator at $37^{\circ} \mathrm{C}$. Phorbol-12-myristate-13-acetate (PMA; Beijing 4A Biotech Co., Ltd) was added to THP-1 monocytes to a final concentration of $40 \mathrm{ng} / \mathrm{mL}$. Approximately $36 \mathrm{~h}$ later, the cell morphology was observed under an inverted microscope to confirm that the THP-1 monocytes had been induced to differentiate into macrophages. Then, IL4 and IL13 (PeproTech, USA) were in turn added to macrophages to a final concentration of $8 \mathrm{ng} / \mathrm{mL}$ and $4 \mathrm{ng} / \mathrm{mL}$, respectively. Approximately $48 \mathrm{~h}$ later, the cell morphology was observed under an inverted microscope to confirm that the macrophages had been induced to differentiate into M2 macrophages. The EC109/9706 cells and M2 macrophages were in turn cultured in the upper and lower chamber of 6-well plates (Corning Inc., Corning, NY, USA). $2 \mathrm{~mL}$ of culture medium were added to the upper and lower chambers, respectively. The cells were placed in an incubator for $48 \mathrm{~h}$ to establish a non-contact coculture. Simultaneously, established the non-contact coculture of EC109/9706 cells and macrophages in the same way.

\subsection{Antibody chip detection}

We used the Human XL Cytokine Array Kit (R \& D; Cat ARY022B) to detect the expression of various factors in cell co-culture samples. For each Array unit, 200-500 $\mu \mathrm{g}$ cell culture supernatant. Each membrane requires $2 \mathrm{ml}$ of Array Buffer to block the protein chip and incubate for 1 hour on a shaker. For each Array unit, it is necessary to dilute the sample with Array Buffer to a volume of $1.5 \mathrm{ml}$ per membrane and incubate with the Array protein membrane at $4^{\circ} \mathrm{C}$ overnight. Wash buffer was used to wash the membrane three times, add diluted detection antibody and incubate at room temperature for 1 hour. Wash the membrane, add streptavidin-HRP, incubate for $30 \mathrm{~min}$, shake at room temperature. Wash the membrane, add $1 \mathrm{ml}$ of chemical color reagent to each well, and develop color on the chemiluminescence imager. Finally, we used professional software to process and analyze the data.

\subsection{DEGs analysis in M1 macrophage and M2 macrophage samples}

DAVID [36] was used to perform analyses of KEGG pathways, GO (CC, BP and MF) and the rank criterion was an FDR $<0.05$. The most important module in the PPI network was built via String online database (http://strin g-db.org Version:11.0) [37]. Herein, a comprehensive Gt score $>0.7$ was considered statistically significant. In order to reveal closely connected regions, we used the Cytoscape [38] to cluster the resulting network.

\section{Results}

\subsection{Identification and analysis of DEGs in macrophages of different phenotypes}


Our previous research showed that a high density M2 macrophage in the TME is closely associated with poor prognosis of ESCC patients and promotes the occurrence and progression of ESCC [6]. Therefore, in order to explore the mechanisms related to tumour occurrence and development, we sought to identify the DEGs in macrophages of different phenotypes. As shown in the Venn diagram, we found that 25 overlapping DEGs were identified in macrophages and M2 macrophages from three datasets (GSE57614, GSE36537, GSE5099) (Fig. 2a). Moreover, 91 overlapping DEGs were shared in M1 and M2 macrophages based on data from four datasets (GSE57614, GSE36537, GSE5099, and GSE95405) (Fig. 2b).

Coincidentally, all 25 DEGs were highly expressed in samples of M2 macrophages. Quantification of the expression of the 25 DEGs in TCGA ESCA using cBioPortal revealed that some of these genes are highly expressed in ESCA tissue (Fig. 2c). A volcano plot revealed that TNFRSF11A expression is highest and CXCL10 is lowest in M2 macrophages (Fig. 2d).

\subsection{FGL2 expression correlates with level of immune cell infiltration in esophageal carcinoma}

To further investigate whether DEGs expression correlated with prognosis of esophageal cancer patients, we used the Kaplan-Meier Plotter online platform to draw survival curves of the 25 DEGs. This analysis revealed that FGL2, ERI1 and WNT5B are related to overall survival (OS) and relapse-free survival (RFS) in ESCC. Notably, FGL2 expression significantly impacts prognosis in ESCC, with high FGL2 expression marginally associated with poorer prognosis $(\mathrm{OS} \mathrm{HR}=2.57,95 \% \mathrm{Cl}=1.05$ to $6.28, \mathrm{P}=0.033$; RFS HR= $3.78,95 \% \mathrm{Cl}=1.43$ to $9.98, \mathrm{P}=0.0039$ ) (Fig. 3a-3b). However, low ERI1 expression was associated with poor prognosis (OS HR $=0.22,95 \% \mathrm{Cl}=0.08$ to $0.59, \mathrm{P}=0.0011$; RFS HR $=0.32,95 \% \mathrm{Cl}=0.12$ to $0.84, \mathrm{P}=$ 0.015) (Fig. 3c-3d). Likewise, low WNT5B expression was associated with poor prognosis (OS HR $=0.41$, $95 \% \mathrm{Cl}=0.19$ to $0.93, \mathrm{P}=0.027$; RFS HR $=4.32,95 \% \mathrm{Cl}=0.99$ to $18.93, \mathrm{P}=0.034$ ) (Fig. 3e-3f). For more information about the functional roles of FGL2, ERI1 and WNT5B, see Table 1.

Table 1

Functional roles of FGL2, ERI1 and WNT5B.

$\begin{aligned} & \text { Gene } \\ & \text { symbol }\end{aligned}$ Full name Function

FGL2 Fibrinogen-like 2 This protein was cloned from cytotoxic T lymphocytes and showed $36 \%$ homology to fibrinogen $\beta$ and $\gamma$ chains, a member of the fibrinogen super family. It is a pleiotropic cytokine that impacts diverse cellular functions.

ERI1 Exoribonuclease

RNA exonuclease that binds to the 3 '-end of histone mRNAs and degrades them, suggesting that it plays an essential role in histone mRNA decay after replication. Also able to degrade the 3 '-overhangs of short interfering RNAs (siRNAs) in vitro, suggesting a possible role as regulator of RNA interference (RNAi).

WNT5B Wnt-family member 5B

This gene is a member of the WNT gene family. It encodes a protein which shows $94 \%$ and $80 \%$ amino acid identity to the mouse Wnt5B protein and the human WNT5A protein, respectively. These proteins have been implicated in oncogenesis and in several developmental processes. 
These results led us to examine why highly expressed genes in M2 macrophages are closely linked with poor prognosis in ESCA and the potential important role they play in regulating TIICs in the TME. Thus, we assessed whether correlations exist between FGL2, ERI1 and WNT5B expression and immune cell infiltration levels in ESCA using TIMER. Tumour purity is a significant factor that influences the genomic analysis of immune cell infiltration in clinical tumour samples [39]. The results show that FGL2 expression was significantly negatively correlated to ESCA tumour purity with significant positive correlations with infiltrating levels of $B$ cells $(r=0.331, P=5.85 e-06), C D 8+T$ cells $(r=0.147, P=4.93 e-$ 02), $C D 4+T$ cells $(r=0.321, P=1.07 e-05)$, macrophages $(r=0.559, P=3.37 e-16)$, neutrophils $(r=0.337, P$

$=3.66 \mathrm{e}-06)$ and dendritic cells $(r=0.268, P=2.73 \mathrm{e}-04)$ (Fig. 4a). However, ERI1 expression did not exhibit significant correlation with ESCA tumour purity, and only correlated weakly with $B$ cells $(r=0.243, P=$ 10.4e-03), CD8 + T cells $(r=0.179, P=1.59 e-02)$ and dendritic cells $(r=0.185, P=1.28 e-02)(F i g .4 b)$. Similarly, WNT5B expression did not correlate significantly with ESCA tumour purity, showing only a weak correlation with macrophages $(r=0.239, P=1.21 e-03)$ (Fig. 4c). These findings strongly suggest that FGL2 plays a specific role in immune cell infiltration in ESCA, especially macrophages.

\subsection{Correlation analysis between FGL2 expression and immune cell marker sets}

To explore the relationship between FGL2 and the various immune infiltrating cells, we focused on examining the correlations between FGL2 expression and various immune cell markers, such as monocytes, TAMs, M1 and M2 macrophages, B cells, T cells (general), CD8 + T cells, neutrophils, NK cells and DCs, in ESCA using the TIMER and GEPIA databases. We also evaluated the different functional T cells, including Tregs, exhausted T cells, Th1, Th2, Tfh and Th17 cells in ESCA (Table 2). After adjusting for purity, we found that the FGL2 expression level was significantly correlated with most immune cells markers and different T cells in ESCA (Table 2). 
Table 2

Correlation analysis between FGL2 and related immune cell genes and markers using TIMER.

Description

\begin{tabular}{|c|c|c|c|c|c|}
\hline & & \multicolumn{2}{|l|}{ None } & \multicolumn{2}{|l|}{ Purity } \\
\hline & & Cor & $P$ & Cor & $P$ \\
\hline \multirow[t]{3}{*}{ Monocyte } & CD86 & 0.683 & 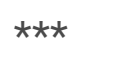 & 0.637 & $\star * \star$ \\
\hline & CD115 (CSF1R) & 0.779 & 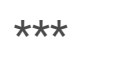 & 0.752 & $\star \star \star$ \\
\hline & CD14 & 0.553 & $\star \star \star *$ & 0.489 & $\star \star \star \star$ \\
\hline \multirow[t]{3}{*}{ TAM } & CD68 & 0.328 & $\star \star *$ & 0.269 & $\star *$ \\
\hline & IL10 & 0.522 & $\star \star *$ & 0.479 & $\star \star \star *$ \\
\hline & CCL2 & 0.578 & $\star \star \star *$ & 0.532 & $\star \star \star *$ \\
\hline \multirow[t]{3}{*}{ M1 Macrophage } & IRF5 & 0.058 & 0.431 & 0.015 & 0.84 \\
\hline & INOS (NOS2) & -0.06 & 0.419 & -0.029 & 0.696 \\
\hline & COX2 (PTGS2) & 0.13 & 0.03 & 0.135 & 0.071 \\
\hline \multirow[t]{3}{*}{ M2 Macrophage } & CD163 & 0.66 & $\star \star \star$ & 0.616 & $\star \star \star \star$ \\
\hline & VSIG4 & 0.647 & $\star \star \star *$ & 0.604 & $\star \star \star$ \\
\hline & MS4A6A & 0.727 & $\star \star \star$ & 0.687 & $\star \star \star$ \\
\hline \multirow[t]{4}{*}{ Treg } & FOXP3 & 0.663 & $\star \star \star *$ & 0.621 & $\star \star \star$ \\
\hline & CCR8 & 0.662 & $\star \star \star$ & 0.622 & $\star \star \star \star$ \\
\hline & STAT5B & 0.395 & $\star \star \star$ & 0.431 & $\star \star \star \star$ \\
\hline & CD4 & 0.778 & $\star \star \star *$ & 0.744 & $\star \star \star$ \\
\hline \multirow[t]{5}{*}{ T cell exhaustion } & PD-1 (PDCD1) & 0.647 & $\star \star *$ & 0.597 & $\star \star \star$ \\
\hline & CTLA4 & 0.646 & $\star \star \star *$ & 0.588 & $\star \star \star$ \\
\hline & LAG3 & 0.602 & $\star \star \star$ & 0.554 & $\star \star \star$ \\
\hline & TIM-3(HAVCR2) & 0.753 & $\star \star \star$ & 0.718 & $\star \star \star$ \\
\hline & GZMB & 0.531 & $\star \star \star$ & 0.462 & $\star \star \star$ \\
\hline CD8 $+\mathrm{T}$ cell & CD8A & 0.634 & 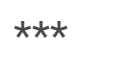 & 0.585 & $\star \star \star *$ \\
\hline
\end{tabular}

Th, T helper cell; Tfh, T follicular helper cell; Treg, T regulatory cell; Cor, R value of Spearman's correlation; None, correlation without adjustment. Purity, correlation adjusted by purity.

$\star P<0.01 ; * \star P<0.001 ; * \star \star P<0.0001$ 


\begin{tabular}{|c|c|c|c|c|c|}
\hline \multirow[t]{2}{*}{ Description } & \multirow{2}{*}{$\begin{array}{l}\text { Gene markers } \\
\text { CD8B }\end{array}$} & \multicolumn{4}{|l|}{ ESCA } \\
\hline & & 0.583 & $\star \star \star *$ & 0.54 & $\star * *$ \\
\hline \multirow[t]{3}{*}{ T cell (general) } & CD3D & 0.609 & $\star \star \star \star ~$ & 0.54 & $\star \star \star *$ \\
\hline & CD3E & 0.642 & $\star \star * *$ & 0.574 & $\star * *$ \\
\hline & $\mathrm{CD} 2$ & 0.662 & $\star \star \star ~$ & 0.606 & $\star \star *$ \\
\hline \multirow[t]{2}{*}{ B cell } & CD19 & 0.356 & $\star \star \star ~$ & 0.248 & ** \\
\hline & CD79A & 0.427 & $\star \star \star$ & 0.333 & $\star * \star$ \\
\hline \multirow[t]{3}{*}{ Neutrophils } & CD66b(CEACAM8) & 0.028 & 0.707 & -0.008 & 0.91 \\
\hline & CD11b (ITGAM) & 0.488 & $\star \star \star$ & 0.421 & $\star \star \star$ \\
\hline & CCR7 & 0.515 & 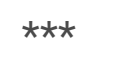 & 0.435 & $\star \star \star *$ \\
\hline \multirow[t]{7}{*}{ Natural killer cell } & KIR2DL1 & 0.271 & $\star \star$ & 0.192 & * \\
\hline & KIR2DL3 & 0.302 & $\star \star \star \star$ & 0.278 & ** \\
\hline & KIR2DL4 & 0.394 & $\star \star \star$ & 0.342 & $\star \star \star *$ \\
\hline & KIR3DL1 & 0.281 & ** & 0.224 & * \\
\hline & KIR3DL2 & 0.22 & * & 0.153 & 0.039 \\
\hline & KIR3DL3 & -0.04 & 0.59 & -0.041 & 0.581 \\
\hline & KIR2DS4 & 0.2 & * & 0.183 & 0.014 \\
\hline \multirow[t]{7}{*}{ Dendritic cell } & HLA-DPB1 & 0.688 & 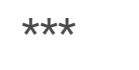 & 0.635 & 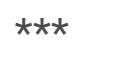 \\
\hline & HLA-DQB1 & 0.424 & 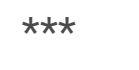 & 0.35 & 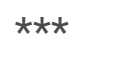 \\
\hline & HLA-DRA & 0.604 & 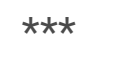 & 0.548 & $\star \star \star *$ \\
\hline & HLA-DPA1 & 0.668 & 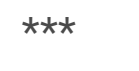 & 0.623 & $\star \star * *$ \\
\hline & $\mathrm{BDCA}-1$ (CD1C) & 0.576 & $\star \star \star \star$ & 0.499 & $* \star *$ \\
\hline & BDCA-4(NRP1) & 0.624 & $\star \star \star \star$ & 0.595 & $\star \star \star *$ \\
\hline & CD11c (ITGAX) & 0.626 & $\star \star \star \star$ & 0.55 & $\star \star *$ \\
\hline \multirow[t]{2}{*}{ Th1 } & T-bet (TBX21) & 0.638 & $\star \star \star$ & 0.568 & $\star \star *$ \\
\hline & STAT4 & 0.672 & 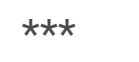 & 0.61 & $\star \star \star *$ \\
\hline
\end{tabular}

Th, T helper cell; Tfh, T follicular helper cell; Treg, T regulatory cell; Cor, R value of Spearman's correlation; None, correlation without adjustment. Purity, correlation adjusted by purity.

${ }^{*} \mathrm{P}<0.01 ;{ }^{* *} \mathrm{P}<0.001 ;$ *** $\mathrm{P}<0.0001$ 


\begin{tabular}{|c|c|c|c|c|c|}
\hline \multirow[t]{4}{*}{ Description } & \multirow{2}{*}{$\begin{array}{l}\text { Gene markers } \\
\text { STAT1 }\end{array}$} & \multicolumn{4}{|l|}{ ESCA } \\
\hline & & 0.437 & $\star \star \star *$ & 0.399 & $\star \star \star *$ \\
\hline & IFN-y (IFNG) & 0.519 & $\star \star \star$ & 0.462 & $\star \star \star *$ \\
\hline & TNF-a (TNF) & 0.165 & 0.025 & 0.106 & 0.155 \\
\hline \multirow[t]{6}{*}{ Th2 } & GATA3 & 0.372 & $\star \star \star$ & 0.311 & $\star \star \star$ \\
\hline & STAT6 & 0.081 & 0.273 & 0.113 & 0.131 \\
\hline & IL13 & 0.324 & $\star \star \star *$ & 0.245 & ** \\
\hline & STAT5A & 0.405 & $\star \star \star *$ & 0.37 & $\star * \star$ \\
\hline & IL2RA (CD25) & 0.649 & $\star \star \star \star$ & 0.606 & $\star \star \star$ \\
\hline & IL2RB (CD122) & 0.687 & $\star \star \star \star$ & 0.648 & $\star \star \star$ \\
\hline \multirow[t]{2}{*}{ Tfh } & BCL6 & 0.197 & * & 0.2 & * \\
\hline & IL21 & 0.252 & ** & 0.188 & 0.012 \\
\hline \multirow[t]{3}{*}{ Th17 } & STAT3 & 0.226 & * & 0.203 & * \\
\hline & IL17A & -0.009 & 0.907 & -0.008 & 0.919 \\
\hline & IL6 & 0.352 & $\star \star \star$ & 0.307 & $\star \star \star$ \\
\hline \multicolumn{6}{|c|}{$\begin{array}{l}\text { Th, T helper cell; Tfh, T follicular helper cell; Treg, T regulatory cell; Cor, R value of Spearman's } \\
\text { correlation; None, correlation without adjustment. Purity, correlation adjusted by purity. }\end{array}$} \\
\hline \multicolumn{6}{|c|}{$\star P<0.01 ; * \star P<<0.001 ; * \star \star x<0.0001}$. \\
\hline
\end{tabular}

Scatterplots of the correlations between FGL2 expression and markers of monocytes (e.g., CD86, CSF1R and CD14) and different macrophage phenotypes (e.g., CD68, CCL2, and IL10 of TAMs, IRF5, NOS2, and PTGS2 of M1 macrophages, CD163, VSIG4, and MS4A4A of M2 macrophages) are shown in Fig. 5.

Interestingly, these results from the TIMER database demonstrate that the expression levels of all markers set of monocytes, TAMs and M2 macrophages correlate strongly with FGL2 expression (Fig. 5a, 5b and 5d). However, M1 macrophage markers did not correlate with FGL2 expression in ESCA (Fig. 5c). These data verify the overlapping results from the GEO database, indicating that the process of TAMs polarization into M2 macrophages may be accompanied by high FGL2 expression. Similar correlation results between FGL2 expression and markers of monocytes, TAMs and M2 macrophages were found using the GEPIA database (Table 3). These findings indicate that FGL2 may regulate TAMs polarization into M2 macrophages in ESCA. 
Table 3

Correlation analysis between FGL2 and markers of monocyte and different phenotypes macrophages in GEPIA.

\begin{tabular}{|c|c|c|c|c|c|}
\hline \multirow[t]{3}{*}{ Description } & \multirow[t]{3}{*}{ Gene markers } & \multicolumn{4}{|l|}{ ESCA } \\
\hline & & \multicolumn{2}{|l|}{ Tumor } & \multicolumn{2}{|c|}{ Normal } \\
\hline & & $\mathrm{R}$ & $P$ & $\mathrm{R}$ & $P$ \\
\hline \multirow[t]{3}{*}{ Monocyte } & CD86 & 0.68 & $\star \star *$ & 0.65 & 0.017 \\
\hline & CD115 (CSF1R) & 0.77 & $\star \star \star \star$ & 0.58 & 0.043 \\
\hline & CD14 & 0.54 & $\star \star \star \star$ & 0.74 & * \\
\hline \multirow[t]{3}{*}{ TAM } & CD68 & 0.3 & $\star \star \star \star$ & 0.42 & 0.15 \\
\hline & IL10 & 0.53 & $\star \star \star \star$ & 0.82 & ** \\
\hline & CCL2 & 0.58 & $\star \star \star \star$ & 0.82 & * \\
\hline \multirow[t]{3}{*}{ M1 Macrophage } & IRF5 & 0.029 & 0.7 & 0.33 & 0.27 \\
\hline & INOS (NOS2) & -0.066 & 0.38 & 0.42 & 0.16 \\
\hline & COX2 (PTGS2) & 0.16 & 0.028 & 0.75 & * \\
\hline \multirow[t]{3}{*}{ M2 Macrophage } & CD163 & 0.63 & $\star * \star$ & 0.87 & ** \\
\hline & VSIG4 & 0.64 & $\star * \star$ & 0.8 & * \\
\hline & MS4A6A & 0.72 & $\star * *$ & 0.81 & * \\
\hline \multicolumn{6}{|c|}{$\begin{array}{l}\text { Tumour, correlation analysis in tumour tissue of TCG; Normal, correlation analysis in normal tissue } \\
\text { TCGA. }\end{array}$} \\
\hline \multicolumn{6}{|c|}{$\star P<0.01 ; * \star P<0.001 ; * \star \star * P<0.0001}$. \\
\hline
\end{tabular}

High FGL2 expression in M2 Macrophages is related to high levels of infiltration by various immune cells in ESCA, especially Tregs, exhausted T cells, DCs and CD8 + T cells. DCs can promote tumour metastasis by reducing CD8 $+T$ cell cytotoxicity and increasing Treg numbers [40]. We also found significant correlations between FGL2 and markers of exhausted T cells and Tregs, such as PD-1, CTLA4, LAG3, TIM3, FOXP3, CCR8 and STAT5B (Table 2). Therefore, these results further confirm that FGL2 is specifically correlated with immune infiltrating cells in ESCA, suggesting that M2 macrophages may regulate immune infiltrating cells by secreting the immunosuppressive factor FGL2 thereby producing a microenvironment that is conducive to tumour growth and which ultimately induces the occurrence and progression of ESCA.

\subsection{FGL2 may promote the initiation and progression of esophageal carcinoma}


We analysed mRNA sequencing data from 184 ESCA patients using the function module of LinkedOmics. The results demonstrate that 4,078 genes showed significant positive correlations with FGL2, whereas 1,177 genes showed significant negative correlations in the volcano plot (Fig. 6a, false discovery rate $[F D R]<0.01)$. The 50 most significant gene sets that correlated positively and negatively with FGL2 are shown in the heatmap (Fig. 5b-5c). These results indicate the widespread impact of FGL2 on the transcriptome.

FGL2 expression is reportedly upregulated in different cancers [41]. Surprisingly, there no statistically significant difference in FGL2 expression was observed between normal and tumour tissues of ESCA (Supplementary Fig. 1). Thus, to better understand whether FGL2 is a crucial factor that mediates the TME and tumour metastasis, we examined cytokines that exhibited significantly different expression levels in in vitro co-culture of macrophages and ESCC cells (Fig. 7a). We found that a $20 \%$ change in cytokine expression between groups was considered statistically significant. Compared with EC109/9706 culture alone or co-culture of macrophages and EC109/9706, we found that IL-10, MMP9, CCL5, TIM3, IL13, VCAM1, M-CSF and FGF-7 expression levels were significantly up-regulated in the M2 macrophage and EC109/9706 co-culture samples (Fig. 7b-7e). Our previous research showed that MMP9 promotes the invasion and migration of ESCA [42]. TAMs polarised into M2 macrophages by cytokines such as macrophage colony-stimulating factor (CSF1, M-CSF), IL-10, IL-13 and IL-4 produce anti-inflammatory cytokines such as TGF- $\beta$ and IL-10 [43]. CCL5 and vascular cell adhesion molecule 1 (VCAM1) promote tumour progression and metastasis in ESCC $[44,45]$. Moreover, fibroblast growth factor-7 (FGF-7) regulates cell migration and invasion through activation of NF-KB transcription factors [46]. These results indicate that M2 macrophages are involved in the secretion of these cytokines in the TME, thereby mediating the occurrence and progression of esophageal carcinoma.

We hypothesized that the immunomodulatory activity of FGL2 plays an important role in the pathogenesis of ESCA. Thus, we investigated whether M2 macrophages regulate the TME by increasing FGL2 expression in ESCA. Next, we evaluated the correlation between FGL2 expression and cytokines, including IL-10, MMP9, CCL5, TIM3, IL-13, VCAM1, M-CSF and FGF-7, in ESCA through cBioPortal. Interestingly, scatterplots show that FGL2 expression correlates specifically with these cytokines and that its expression is strongly positively related to all of them (Fig. 8a-7 h). In addition, TIM-3 (HAVCR2) [47], as a crucial gene that regulates $T$ cell exhaustion, showed a significant positive correlation with FGL2 expression, indicating that high FGL2 expression plays an important role in TIM-3-mediated T cell exhaustion. FOXP3 also plays an important role in Tregs, leading to the suppression of cytotoxic T cells attack of tumour cells [48]. A strong, positive correlation was found between PD-1 and CTLA-4 in T cell exhaustion (Supplementary Fig. 2), which is consistent with the results in Table 2. These results further confirm the findings that M2 macrophages regulate the TME via increased FGL2 expression in ESCA. In addition, FGL2 expression was specifically correlated with the presence of immune infiltrating cells in ESCA, suggesting that FGL2 plays a vital role in immune escape and immunosuppression in the esophageal cancer microenvironment. 


\subsection{Reversing TAM polarization may provide new targets for tumour immunotherapy}

Our previous study showed that TAMs contribute directly to the survival, invasion and metastasis of esophageal cancer cells [49]. For the purpose of finding a potential effective immunotherapy target, we conducted an enrichment analysis of GO biological functions (Biological Process, Cellular Component and Molecular Function) and KEGG pathways of the 91 DEGs via DAVID. The results from this GO analysis indicate that the DEGs were primarily enriched for the following: Type I interferon signalling pathway, Response to interferon-beta, Pyroptosis, Protein polyubiquitination, Positive regulation of interleukin-1 beta secretion, NLRP3 inflammasome complex, Interferon-gamma-mediated signalling pathway, Innate immune response, Defence response to virus and AIM2 inflammasome complex (Fig. 9a). Furthermore, KEGG pathway enrichment analysis demonstrated that these DEGs were enriched for pathways involved in: the Toll-Like receptor signalling pathway, RIG-I-like receptor signalling pathway, NF-KB signalling pathway, Measles, Influenza A, Herpes simplex infection, Hepatitis C, Hepatitis B, Cytosolic DNA-sensing pathway and Cytokine-cytokine receptor interaction (Fig. 9b). These results suggest that activation of M1 macrophages up-regulate gene pathways and that blocking them may produce beneficial effects that improve anti-tumour immunity.

We also analysed the functional interaction between proteins to understand the underlying mechanisms leading to tumour occurrence and development. STRING database screening and the Cytoscape software were used to visualize closely connected regions in the PPI network. The MCODE plugin was used to identify the top hub genes, which constituted the most closely connected module. The PPI network includes 54 nodes and 422 edges (Fig. 9c). The top 30 genes out of the 54 hub genes are shown in Fig. 9d. These key genes provide new insight for developing novel molecular drugs that target TAMs in immunotherapies for treating esophageal carcinoma.

Research has shown that FGL2 is a potential molecular target for glioblastoma treatment [50]. Therefore, FGL2 gene set enrichment analysis (GSEA) was used to identify the functions or pathway activity variations involved in ESCA. This analysis revealed enrichment of the MAPK, JAK/STAT, Toll-Like Receptors (TLRs) and other immune-related pathways (Supplementary Fig. 3). A study showed that the presence of FGL2 in tumour cells inhibited granulocyte-macrophage colony-stimulating factor (GM-CSF)induced CD103 + DC differentiation by suppressing STAT1/5 and p38 activation [51]. Liu, et al. also reported that MAPK-mediated up-regulation of FGL2 promotes proliferation, migration and invasion of colorectal cancer cells [52]. A schematic diagram depicting the potential role of FGL2 in esophageal carcinoma is shown in Fig. 10. Taken together, these results indicate that targeting FGL2 may potentially weaken the immunosuppressive activity of the tumour and thus inhibit its progression.

\section{Discussion}

Macrophages constitute a key immune population that can prevent or promote tumour growth in the TME. The unique macrophage subtype, M2-like TAMs, can suppress anti-tumour immunity, induce 
angiogenesis and promote cell migration [53]. The number of M2-like TAMs increases with tumour progression, and has been closely linked with poor patient prognosis [54]. This result is consistent with our previous findings in esophageal cancer. Furthermore, TAMs are an important component in the esophageal cancer microenvironment and the increase in TAMs frequency is closely related to the high invasiveness and poor prognosis seen in ESCA patients [49]. Increased FGL2 expression has been detected in several human tumours, such as gliomas [55], lymphomas [56], lung [57] and liver cancer [58]. To our knowledge, this research is the first to show the correlation between high FGL2 expression in M2 macrophages and poor prognosis of OS and RFS in esophageal cancer patients. In our present study, we found that, while genetic markers of M1 macrophages (IRF5, INOS and COX2) were not correlated with FGL2 expression, genetic markers of M2 macrophages (CD163, VSIG4 and MS4A6A) showed strong correlation with FGL2 expression. These results indicate that M2 macrophages are highly likely to mediate the initiation and progression of esophageal carcinoma by secreting the immunosuppressive factor FGL2 to regulate the TME. Moreover, our data revealed the potential regulatory role of FGL2 in the polarization of TAMs.

There is a significant correlation between FGL2 expression and genetic markers of T cell exhaustion, Tregs, DCs and CD8 + T cells in ESCA. In addition, correlations can be found between FGL2 expression and the regulation of several genetic markers of T helper cells (Th1, Th2, Tfh and Th17) in ESCA. These studies have demonstrated that exposure of immature DCs to soluble FGL2 inhibits the expression of MHC II molecules and CD80, thereby inhibiting DC maturation and antigen presentation capabilities [59]. The binding of FGL2 to FcyRIIB has been reported to hinder DC and B cell activity [60]. These associations could be indicative of a potential mechanism where FGL2 regulates immune infiltrating cell functions in ESCA. Analysis of co-expressed genes illustrated that cysteinyl leukotriene receptor 1 (CYSLTR1, the receptor for LTD4) and MS4A6A correlate positively with FGL2 expression. The study shows that MMP-9 activity and expression were reduced by a CYSLTR1 antagonist called montelukast, and that M2 macrophages enriched in LTD4 promote the migration and invasion of colon cancer cells via MMP-9 expression [61]. In addition, MS4A4A and MS4A6A have been closely linked to the poor prognosis observed in ovarian cancer, and that CD163, MS4A4A and MS4A6A serve as surface markers for M2 macrophages [62]. Collectively, these results are similar to the present study. We found that FGL2 expression correlated positively with up-regulated IL-10, MMP9, CCL5, TIM3, IL-13, VCAM1, M-CSF and FGF-7 expression in a co-culture of M2 macrophages with EC109/9706. CCL5, CSF-1 and CCL2 are reportedly involved in monocyte recruitment. In particular, CSF-1 is involved in monocyte recruitment, macrophage survival and M2 macrophage polarization [63]. These findings indicate that $M 2$ macrophages may stimulate the expression of cancer-promoting factors in the TME by secreting FGL2, and that elevated FGL2 expression drives the polarization of TAMs towards an M2 phenotype. Together these results demonstrate that FGL2 plays a crucial role in the recruitment and regulation of immune infiltrating cells in ESCA. FGL2 exerts its immunosuppressive effects through various pathways.

FGL2 is mainly expressed by activated endothelial cells, macrophages, T cells and tumour cells. Studies have also shown that FGL2 derived from the tumour matrix could promote the occurrence and development of lung cancer [56]. Interestingly, FGL2 is mainly but not exclusively expressed by immune 
cells, tumour cells and tumour stroma in the TME, and hematopoietic cells can also produce FGL2 [64]. Notably, FGL2 expression in normal tissues is more strongly correlated with TAMs and M2 macrophages than in ESCA tumour tissues (Table 3). Further investigation is required to understand whether FGL2 detection in peripheral blood can be used as a tumour marker for early screening and diagnosis of esophageal carcinoma. In the TME, M2 macrophages secrete FGL2 to exert an immunosuppressive effect, and immune infiltrating cells and tumour cells can also secrete FGL2 to promote the polarization of TAMs into M2 macrophages, forming an important carcinogenic loop that promotes the initiation and progression of esophageal carcinoma. Our results demonstrate that targeting FGL2 may reduce the polarization of $\mathrm{M} 2$ macrophages, weaken immunosuppression and thus inhibit the progression of esophageal carcinoma. These characteristics make FGL2 an important molecule to study for its potential as a therapeutic target and immunomodulator.

The main target of cancer immunotherapy is to reduce immunosuppression or restore and enhance the damaged anti-tumour immunity. Broadly, there are two methods for targeting TAMs and enhancing anticancer efficacy, namely inhibiting the recruitment of TAMs to the tumour site and reprogramming TAMs into anti-tumour M1 macrophages. TAMs express the ligands for checkpoint molecules in T cells, including PD-L1, PD-L2, VISTA and B7H4, which are upregulated in response to various stimuli involving hypoxia and cytokines $[65,66]$. Currently, multiple antibodies and small molecule inhibitors are being evaluated in clinical studies. They can be used as a monotherapy or in combination with standard radiotherapy and chemotherapy. In a pancreatic cancer preclinical model, the inhibitor Ibrutinib of Bruton's tyrosine kinase (BTK) was used to reset macrophages toward an M1-like phenotype that promoted CD8 + T cell cytotoxicity. Currently, this strategy is under evaluation in combination with checkpoint inhibitors [67].

TLRs are expressed on leukocytes such as macrophages and DCs, which induce inflammatory responses by recognizing damaged cell products and pathogen-derived molecules. Several preclinical studies demonstrated that agonists targeting TLRs (TLR3, TLR7/8 and TLR9) in macrophages potentially enhance anti-tumour efficacy. Importantly, this strategy polarizes macrophages into Th1 and M1-like TAMs and inhibit tumour progression [68]. DEGs between M1 and M2 macrophages samples showed that TNFRSF11A, HGSNAT, MCM6, RNASE6, FAM101B, ACPP, EMB and NCAPH were significantly upregulated in $M 2$ macrophages, of which TNFRSF11A expression was the highest. There was a crucial role for TNFRSF11A in cervical cancer cell migration, invasiveness and proliferation has been reported [69]. Targeting these genes may reduce the polarization of TAMs into M2 macrophages and weaken the immunosuppressive effect. GO analysis results showed that the significant DEGs were primarily enriched for the type I interferon signalling pathway. Type I interferon is a cytokine that plays a key role in anti-viral, anti-tumour, and immune regulation. When type I interferon receptor (Interferon-a/ $\beta$ receptor, IFNAR) binds to its ligand, activated IFNAR1 binds to TYK2 (tyrosine kinase 2), inducing IFNAR2 to bind to JAK1 (Janus kinase 1) to activate the JAK/STAT, MAPK and PI3K/mTOR signalling pathways [70]. Crosstalk has also been demonstrated between these different signalling pathways. The activation of these pathways is also closely related to the occurrence, growth, apoptosis inhibition and metastasis of tumour cells. Cytokine-mediated signal transduction in vivo is a complex network system, and related basic research 
work will continue to face challenges for a long time to come. Our analysis of the protein interaction network revealed that most of the closely connected genes were significantly up-regulated in M1 macrophages, including STAT1, OAS1, OAS2 and DDX5. These proteins participate in many important biological function pathways in the TME, so using them to develop small molecule agonists may enhance anti-tumour immunity.

\section{Conclusion}

In summary, the results of this study provide many directions for our follow-up research on related mechanisms involved in the esophageal cancer microenvironment. FGL2, which is highly expressed by M2 macrophages, is expected to become a potential tumour marker for early diagnosis, as well as an effective immunotherapy target for treating esophageal carcinoma. DEGs between M1 and M2 macrophages may also be targeted to reprogram TAMs into anti-tumour M1 macrophages for cancer immunomodulation therapy.

\section{Abbreviations}

ESCA: esophageal carcinoma; GEO: Gene Expression Omnibus; DEGs: differentially expressed genes; KEGG: Kyoto Encyclopedia of Genes and Genomes; TAMs: Tumour-associated macrophages; FGL2: Fibrinogen-like protein 2.

\section{Declarations}

\section{Acknowledgements}

None.

\section{Authors' contributions}

$\mathrm{XY}$ and $\mathrm{JMH}$ designed the study. $\mathrm{XY}, \mathrm{YL}$ and $\mathrm{AZZ}$ collected the literature. $\mathrm{XY}, \mathrm{JMH}$ and $\mathrm{AZZ}$ performed statistical analyses. YL, CHJ, YFX, JFL, FPL, CXL, WHL, LJP, XHS, HJZ, and FL analysed the data. XY wrote the manuscript. All authors read and approved the final manuscript.

\section{Funding}

This work was supported by National Natural Science Foundation of China (N0.81760428, N0.81960435 and N0.81460363), Start-up Project of High-level Talents Scientific Research in Shihezi University (RCZK2018C19), Science and Technology Development Project of Xinjiang Production and Construction Corps (NO. 2018AB033), National Early Detection and Treatment Project for Upper Digestive Tract in Rural Area in China (NO.2018), and The Youth Science and Technology Innovation Leading Talents Project of 
Corps (NO.2017CB004). The funders had no role in study design, data collection and analysis, decision to publish, or preparation of the manuscript.

\section{Availability of data and materials}

The datasets during and/or analyzed during the current study available from the corresponding author on reasonable request.

\section{Ethics approval and consent to participate}

As the data (TCGA and GEO datasets, etc.) are publicly available, no ethical approval is required.

\section{Consent for publication}

Not applicable.

\section{Competing interests}

The authors declare that they have no competing interests.

\section{References}

1. Gupta B, Kumar N. Worldwide incidence, mortality and time trends for cancer of the oesophagus. Eur J Cancer Prev 2017: 107-18.

2. Siegel RL, Miller KD, Jemal A. Cancer statistics, 2019. CA Cancer J Clin 2019: 7-34.

3. Riley R S, June $\mathrm{CH}$, Langer R, et al. Delivery technologies for cancer immunotherapy[J]. Nature Reviews Drug Discovery, 2019.

4. Biswas SK, Mantovani A. Macrophage plasticity and interaction with lymphocyte subsets: cancer as a paradigm. Nat Immunol. 2010:889-896.

5. Wynn TA, Chawla A, Pollard JW. Macrophage biology in development, homeostasis and disease. Nature. 2013:445-455.

6. Jian Ming Hu, Kai Liu, Ji Hong Liu, et al. CD163 as a marker of M2 macrophage, contribute to predict aggressiveness and prognosis of Kazakh esophageal squamous cell carcinoma[J]. Oncotarget, 2017: 21526-21538

7. Wang X L, Liu K, Liu J H, et al. High infiltration of CD68-tumor associated macrophages, predict poor prognosis in Kazakh esophageal cancer patients[J]. International Journal of Clinical \& Experimental Pathology, 2017:10282-10292. 
8. Lee $\mathrm{C}$, Jeong $\mathrm{H}, \mathrm{Bae} \mathrm{Y}$, et al. Targeting of M2-like tumor-associated macrophages with a melittinbased pro-apoptotic peptide[J]. 2019.

9. Petty A J, Yang Y. Tumor-associated macrophages: implications in cancer immunotherapy[J]. Immunotherapy, 2017:289-302.

10. Hu J, Yan J, Rao G, et al. The duality of Fgl2-secreted immune checkpoint regulator versus membrane-associated procoagulant: therapeutic potential and implications. Int Rev Immunol. 2016:325-39.

11. Shalev I, Liu H, Koscik C, et al. Targeted deletion of fgl2 leads to impaired regulatory T cell activity and development of autoimmune glomerulonephritis. J Immunol. 2008:249-60.

12. Melnyk M, Shalev I, Zhang J, et al. The prothrombinase activity of FGL2 contributes to the pathogenesis of experimental arthritis[J]. Scandinavian Journal of Rheumatology, 2011, 40(4):269278.

13. Shalev, I. et al. The novel CD4+ CD25+ regulatory $T$ cell effector molecule fibrinogen-like protein 2 contributes to the outcome of murine fulminant viral hepatitis. Hepatology. 2009:387-397.

14. Foerster, K. et al. The novel immunoregulatory molecule FGL2: a potential biomarker for severity of chronic hepatitis C virus infection. Journal of hepatology. 2010:608-615.

15. Joller N, Lozano E, Burkett PR, Patel B, Xiao S, Zhu C, et al. Treg cells express-ing the coinhibitory molecule TIGIT selectively inhibit proinflammatory Th1 and Th17 cell responses. Immunity. 2014:569-81.

16. Chan CW, Kay LS, Khadaroo RG, Chan MW, Lakatoo S, Y oung KJ, et al. Soluble fibrinogen-like protein 2/fibroleukin exhibits immunosuppressive properties: suppressing $T$ cell proliferation and inhibiting maturation of bone marrow-derived dendritic cells. J Immunol. 2003:4036-44.

17. Ying Z, Longhui Z, Haoran Z, et al. Stroma-derived Fibrinogen-like Protein 2 Activates Cancerassociated Fibroblasts to Promote Tumor Growth in Lung Cancer[J]. International Journal of Biological Sciences. 2017:804-814.

18. Tang M, Cao X, Li P, et al. Increased expression of Fibrinogen-Like Protein 2 is associated with poor prognosis in patients with clear cell renal cell carcinoma[J]. Scientific Reports. 2017:12676.

19. Dwyer, K. M. et al. CD39 and control of cellular immune responses. Purinergic signalling. 2007:171180.

20. Li J Q, Yu X J, Wang Y C, et al. Distinct patterns and prognostic values of tumor-infiltrating macrophages in hepatocellular carcinoma and gastric cancer[J]. Journal of Translational Medicine, 2017:1-11.

21. Derlindati E, Dei Cas A, Montanini B, Spigoni V et al. Transcriptomic analysis of human polarized macrophages: more than one role of alternative activation? PLoS One 2015: e0119751.

22. Mehraj V, Textoris J, Ben Amara A, Ghigo E et al. Monocyte responses in the context of Q fever: from a static polarized model to a kinetic model of activation. J Infect Dis. 2013:942-51. 
23. Martinez FO, Gordon S, Locati M, Mantovani A. Transcriptional profiling of the human monocyte-tomacrophage differentiation and polarization: new molecules and patterns of gene expression. $\mathrm{J}$ Immunol. 2006:7303-11.

24. Solinas G, Schiarea S, Liguori M, Fabbri M et al. Tumor-conditioned macrophages secrete migrationstimulating factor: a new marker for M2-polarization, influencing tumor cell motility. J Immunol. 2010:642-52.

25. Unberath P, Knell C, Prokosch HU, Christoph J. Developing new analysis functions for a translational research platform: extending the cBioPortal for Cancer Genomics. Stud Health Technol Inform. 2019:46-50.

26. Lanczky A, Nagy A, Bottai G, Munkacsy G, Szabo A, Santarpia L, et al. miRpower: a web-tool to validate survival-associated miRNAs utilizing expression data from 2178 breast cancer patients. Breast Cancer Res Treat. 2016:439-46.

27. Li T, Fan J, Wang B, Traugh N, Chen Q, Liu JS, et al. TIMER: a web server for comprehensive analysis of tumor-infiltrating immune cells. Cancer Res. 2017: e108-e110.

28. Li B, Severson E, Pignon JC, Zhao H, Li T, Novak J, et al. Comprehensive analyses of tumor immunity: implications for cancer immunotherapy. Genome Biol. 2016:174.

29. Aran D, Sirota M, Butte AJ. Systematic pan-cancer analysis of tumour purity. Nat Commun. 2015:8971.

30. Siemers NO, Holloway JL, Chang H, Chasalow SD, Ross-MacDonald PB, Voliva CF, et al. Genomewide association analysis identifiesgeneticcorrelates of immune infiltrates in solid tumors. PLoS ONE. 2017: e0179726.

31. Danaher P, Warren S, Dennis L, D'Amico L, White A, Disis ML, et al. Gene expression markers of Tumor Infiltrating Leukocytes. J Immunother Cancer. 2017:18.

32. Sousa S, Maatta J. The role of tumour-associated macrophages in bone metastasis. J Bone Oncol. 2016:135-8.

33. Tang Z, Li C, Kang B, Gao G, Li C, Zhang Z. GEPIA: a web server for cancer and normal gene expression profiling and interactive analyses. Nucleic Acids Res. 2017: W98-w102.

34. Vasaikar SV, Straub P, Wang J, Zhang B. LinkedOmics: analyzing multi-omics data within and across 32 cancer types. Nucleic Acids Res. 2018: D956-D63.

35. Subramanian A, Tamayo P, Mootha VK, et al. Gene set enrichment analysis: a knowledge-based approach for interpreting genome-wide expression profiles. Proc Natl Acad Sci USA. 2005:1554515550

36. Da Huang W, Sherman BT, Lempicki RA. Systematic and integrative analysis of large gene lists using DAVID bioinformatics resources. Nat Protoc. 2009:44-57.

37. Szklarczyk D, Franceschini A, Wyder S, Forslund K, Heller D, Huerta Cepas J, et al. STRING v10: protein-protein interaction networks, integrated over the tree of life. Nucleic Acids Res. 2015: D44752. 
38. Shannon P, Markiel A, Ozier O, Baliga NS, Wang JT, Ramage D, et al. Cytoscape: a software environment for integrated models of biomolecular interaction networks. Genome Res. 2003:2498504.

39. Yoshihara K, Shahmoradgoli M, Martinez E, Vegesna R, Kim H, TorresGarcia W, et al. Inferring tumor purity and stromal and immune cell admixture from expression data. Nat Commun. 2013:2612.

40. Sawant A, Hensel JA, Chanda D, Harris BA, Siegal GP, Maheshwari A, et al. Depletion of plasmacytoid dendritic cellsinhibits tumor growth and prevents bone metastasis of breast cancer cells. J Immunol. 2012:4258-65.

41. Hu, J. et al. The Duality of Fgl2-Secreted Immune Checkpoint Regulator Versus MembraneAssociated Procoagulant: Therapeutic Potential and Implications. International reviews of immunology. 2014:325-339.

42. Li J, Xie Y, Wang X, et al. Overexpression of VEGF-C and MMP-9 predicts poor prognosis in Kazakh patients with esophageal squamous cell carcinoma[J]. PeerJ, 2019:8182.

43. Benner Brooke, Scarberry Luke, Suarez-Kelly Lorena P et al. Generation of monocyte-derived tumorassociated macrophages using tumor-conditioned media provides a novel method to study tumorassociated macrophages in vitro. [J]. Immunother Cancer, 2019: 140.

44. Wu Y C, Shen Y C, Chang W C, et al. Autocrine CCL5 promotes tumor progression in esophageal squamous cell carcinoma in vitro[J]. Cytokine, 2018:94-103.

45. Zhang B, Zhang Z, Li L, et al. TSPAN15 interacts with BTRC to promote oesophageal squamous cell carcinoma metastasis via activating NF-KB signaling[J]. Nature Communications, 2018:1423.

46. Jiangong, Niu, Zhe, et al. Keratinocyte Growth Factor/Fibroblast Growth Factor-7-regulated Cell Migration and Invasion through Activation of NF-KB Transcription Factors. [J]. Journal of Biological Chemistry, 2007.

47. Ruihan Tang, Manu Rangachari, Vijay K Kuchroo. Tim-3: A Co-Receptor With Diverse Roles in T Cell Exhaustion and Tolerance. Semin Immunol. 2019.

48. Foxp3 + T Regulatory Cells: Still Many Unanswered Questions-A Perspective After 20 Years of Study

49. Hu JM, Liu K, Liu JH, et al. The increased number of tumor-associated macrophage is associated with overexpression of VEGF-C, plays an important role in Kazakh ESCC invasion and metastasis [J]. Exp Mol Pathol. 2017:15-21.

50. Patel R, Traylor J I, Latha K, et al. Fibrinogen-like protein 2: a potential molecular target for glioblastoma treatment[J]. Expert Opinion on Therapeutic Targets, 2019.

51. Yan J, Zhao Q, Gabrusiewicz K, et al. FGL2 promotes tumor progression in the CNS by suppressing CD103+ dendritic cell differentiation[J]. Nature Communications, 2019.

52. Liu X, Chu Y, Wang D, et al. MAPK-mediated upregulation of fibrinogen-like protein 2 promotes proliferation, migration and invasion of colorectal cancer cells[J]. Cell Biology International, 2019.

53. Kitamura T, Qian BZ, Pollard JW. Immune cell promotion of metastasis [J]. Nat Rev Immunol. 2015:73-86. 
54. Yang S, Liu T, Cheng Y, Bai Y, Liang G. Immune cell infiltration as a biomarker for the diagnosis and prognosis of digestive system cancer [J]. Cancer Sci. 2019:3639-3649.

55. Khatri, Latha, Jun, et al. The Role of Fibrinogen-Like Protein 2 on Immunosuppression and Malignant Progression in Glioma. [J]. Journal of the National Cancer Institute, 2018.

56. Rabizadeh, E. et al. Increased activity of cell membrane-associated prothrombinase, fibrinogen-like protein 2, in peripheral blood mononuclear cells of B-cell lymphoma patients. PloS one. 2014: e109648.

57. Ying Z, Longhui Z, Haoran Z, et al. Stroma-derived Fibrinogen-like Protein 2 Activates Cancerassociated Fibroblasts to Promote Tumor Growth in Lung Cancer[J]. International Journal of Biological Sciences, 2017:804-814.

58. Su, K. et al. Fibrinogen-like protein $2 /$ fibroleukin prothrombinase contributes to tumor hypercoagulability via IL-2 and IFN-gamma. World journal of gastroenterology. 2008: 5980-5989.

59. Chan CW, Kay LS, Khadaroo RG, et al. Soluble fibrinogen-like protein 2/fibroleukin exhibits immunosuppressive properties: suppressing $T$ cell proliferation and inhibiting maturation of bone marrow-derived dendritic cells. J Immunol. 2003:4036-4044.

60. Luft O, Khattar R, Farrokhi K, et al. Inhibition of the fibrinogen-like protein 2:

FcgammaRIIB/RIllimmunosuppressive pathway enhances antiviral T-cell and B-cell responses leading to clearance of lymphocytic choriomeningitis virus clone 13. Immunology. 2018:476-489.

61. Vinnakota K, Zhang Y, Selvanesan B C, et al. M2-like macrophages induce colon cancer cell invasion via matrix metalloproteinases[J]. Journal of Cellular Physiology, 2017.

62. Katyayni Vinnakota, Yuan Zhang, Benson Chellakkan Selvanesan et al. M2-like Macrophages Induce Colon Cancer Cell Invasion via Matrix Metalloproteinases[J]. J Cell Physiol. 2017:3468-3480.

63. Noy R, Pollard JW. Tumor-Associated Macrophages: From Mechanisms to Therapy. Immunity. 2014:49-61.

64. Ying Z, Jie Z, Yi F, et al. Control of Intestinal Inflammation, Colitis-Associated Tumorigenesis, and Macrophage Polarization by Fibrinogen-Like Protein 2[J]. Frontiers in Immunology, 2018:87.

65 . Wang $L$, et al. VISTA, a novel mouse Ig superfamily ligand that negatively regulates $T$ cell responses. J Exp Med. 2011:577-92.

66. Kryczek I, et al. Cutting edge: induction of B7-H4 on APCs through IL-10: novel suppressive mode for regulatory T cells. J Immunol. 2006:40-4.

67. Alberto Mantovani, Federica Marchesi, Alberto Malesci, Luigi Laghi, Paola Allavena. TumorAssociated Macrophages as Treatment Targets inOncology[J]. Nature Reviews Clinical Oncology, 2017:399.

68. Kumar S, Ramesh A, Kulkarni A. Targeting macrophages: a novel avenue for cancer drug discovery[J]. Expert Opinion on Drug Discovery, 2020.

69. Ma D, Chang L Y, Zhao S, et al. KLF5 promotes cervical cancer proliferation, migration and invasion in a manner partly dependent on TNFRSF11a expression[J]. Scientific Reports, 2017:15683. 
70. Ivashkiv L B, Donlin L T. Regulation of type I interferon responses[J]. Nature Reviews Immunology, 2014.

\section{Figures}

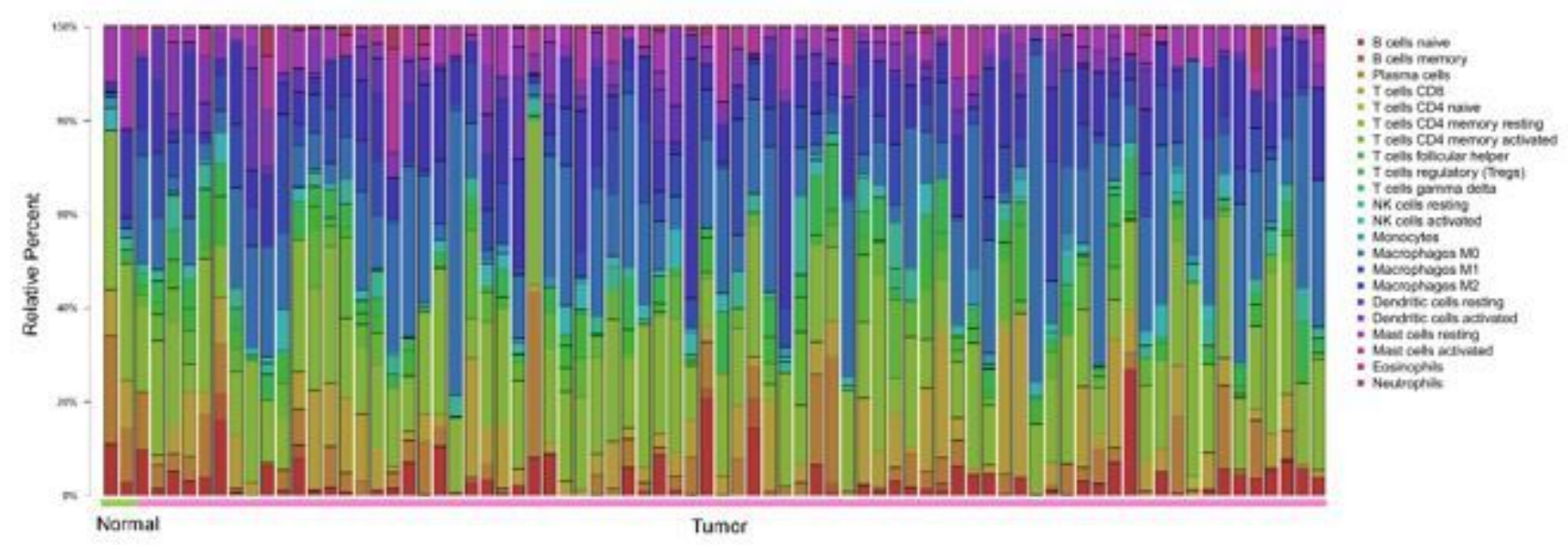

\section{Figure 1}

The landscape of 22 tumour-infiltrating cells in ESCA (normal $=2 \nabla$ tumour $=76$ ) and their proportions in each sample as quantified by CIBERSORT. 

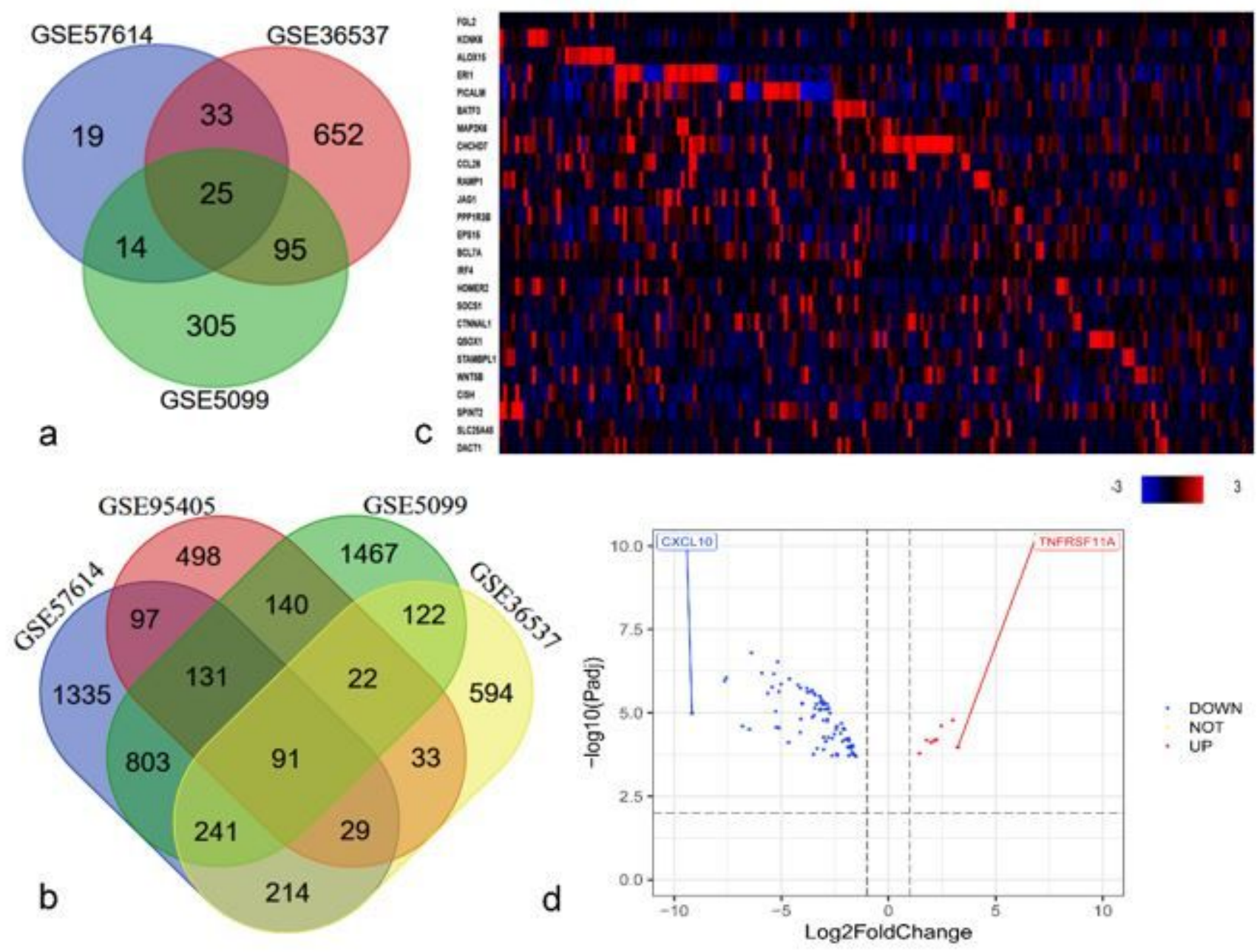

3
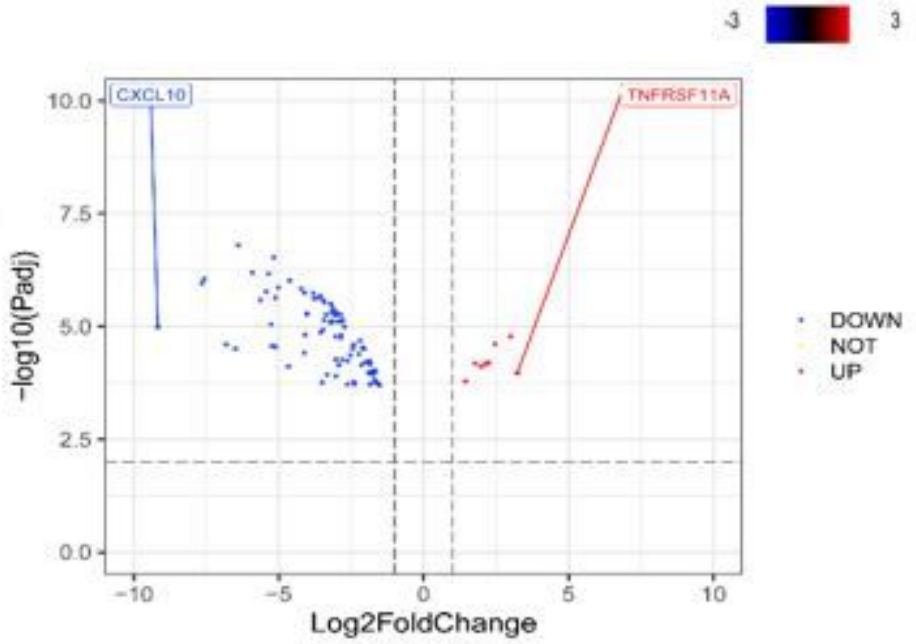

Figure 2

Quantitative analysis of DEGs in macrophages of different phenotypes. a. 25 overlapping DEGs in macrophages and M2 macrophages were identified from three datasets (GSE57614, GSE36537, GSE5099) based on $|\operatorname{logFC}| \geq 1.0$ with an adjusted p-value < 0.05. b. 91 overlapping DEGs in M1 and M2 macrophages were identified from four datasets (GSE57614, GSE36537, GSE5099, and GSE95405) based on $|\log F C| \geq 1.0$ with an adjusted $p$-value $<0.01$. c. Expression of the 25 overlapping DEGs is shown as a heatmap as quantified in TCGA ESCA (tumour $=184$ ) by cBioPortal. High, medium and low expressing genes are represented in red, black, and blue, respectively. d. Volcano plot of the 91 overlapping DEGs. Compared with M1 macrophages, red spots represent eight up-regulated genes and blue spots indicate 83 down-regulated genes in M2 macrophage samples. 

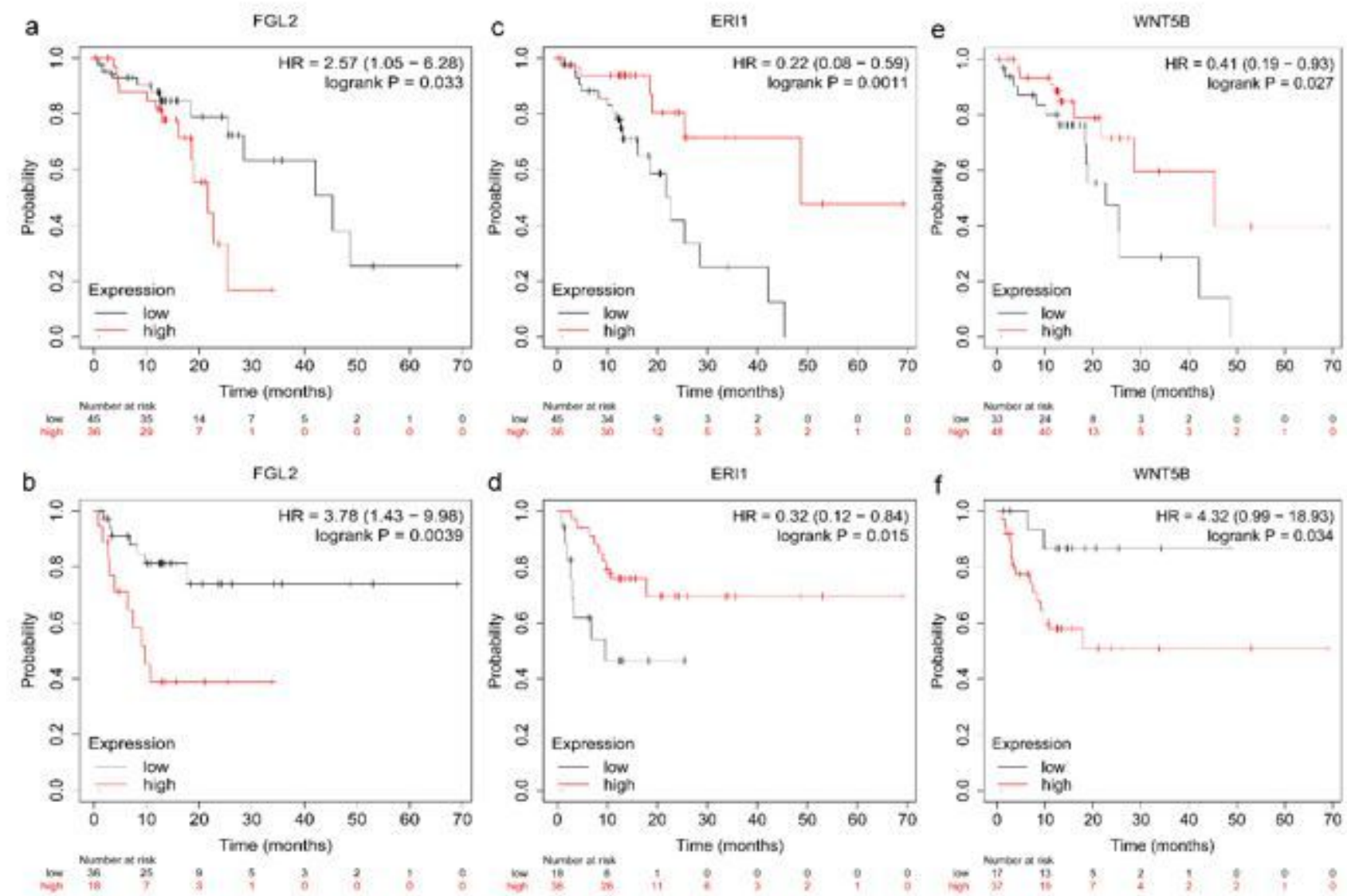

\section{Figure 3}

Kaplan-Meier Plotter online platform survival curves comparing the expression of (a) FGL2 overall survival (OS), (b) FGL2 relapse-free survival (RFS), (c) ERI1 OS, (d) ERI1 RFS, (e) WNT5B OS and (f) WNT5B RFS in ESCC $(n=81)$. $P<0.05$ was considered statistically significant.

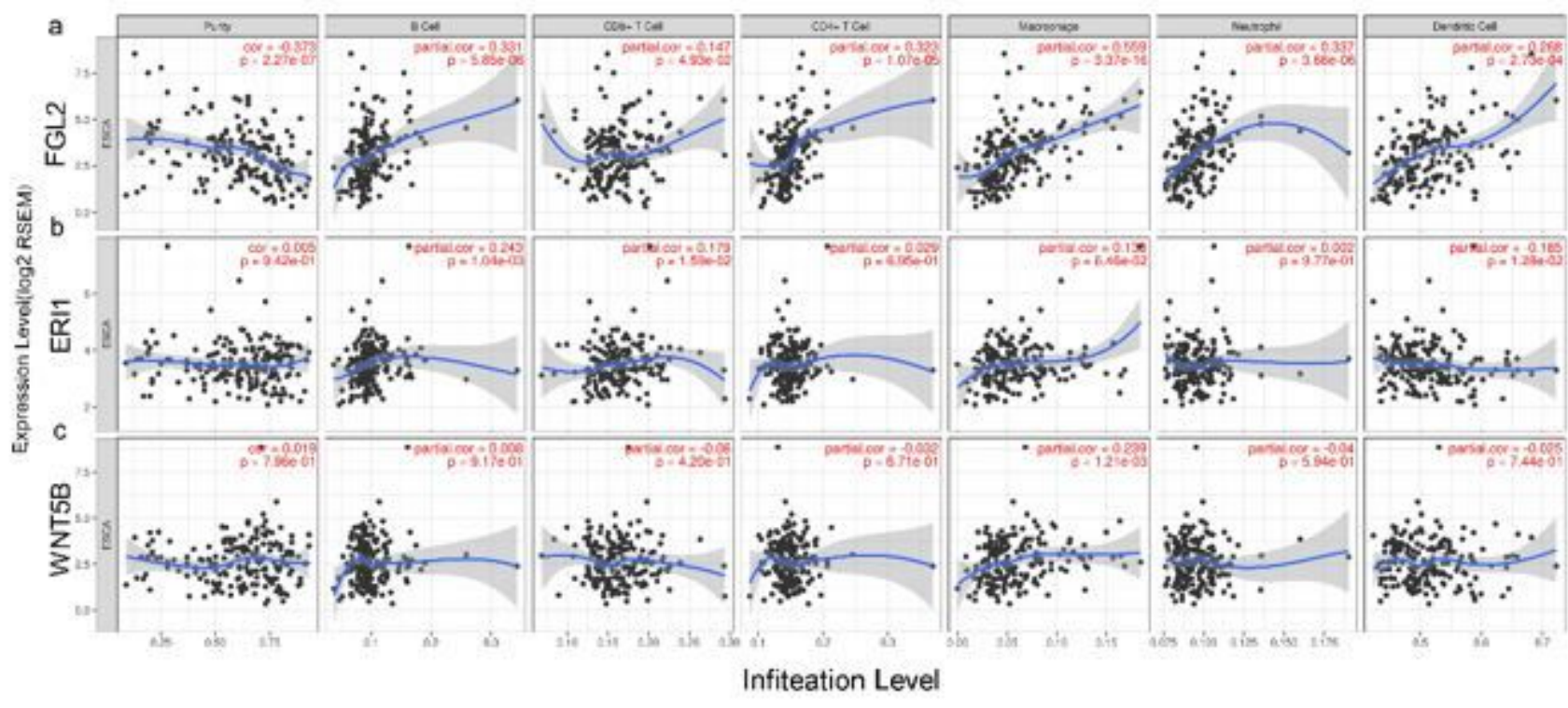




\section{Figure 4}

Correlation of FGL2, ERI1 and WNT5B expression with the level of immune cell infiltration in ESCA. a. FGL2 expression was significantly negatively correlated with ESCA tumour purity, displaying significant positive correlations with infiltrating levels of B cells, CD8+ T cells, CD4+ T cells, macrophages, neutrophils and dendritic cells. b-c. ERI1 and WNT5B expression did not correlate significantly with ESCA tumour purity. ERI1 expression showed a weak correlation with B cells, CD8+ T cells and dendritic cells (b). WNT5B expression showed only a weak correlation with macrophages (c). $\mathrm{P}<0.05$ was considered statistically significant.
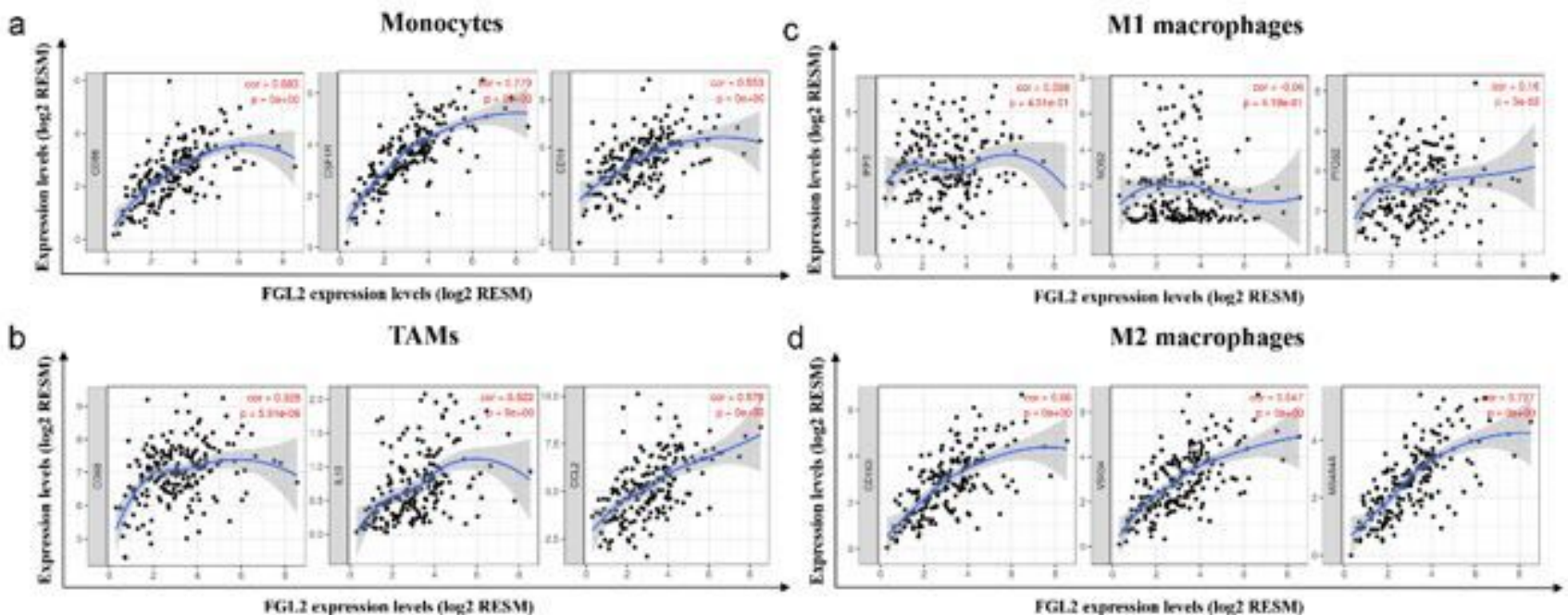

\section{Figure 5}

FGL2 expression correlated with tumour-associated macrophage polarization in ESCA. Immune cell markers assessed include CD86, CSF1R and CD14 of monocytes; CD68, CCL2 and IL10 of TAMs; IRF5, NOS2 and PTGS2 of M1 macrophages; and CD163, VSIG4 and MS4A4A of M2 macrophages. A-d. Scatterplots of correlations between FGL2 expression and genetic markers of monocytes (a), TAMs (b), M1 macrophages (c) and M2 macrophages (d). P $<0.05$ was considered statistically significant.
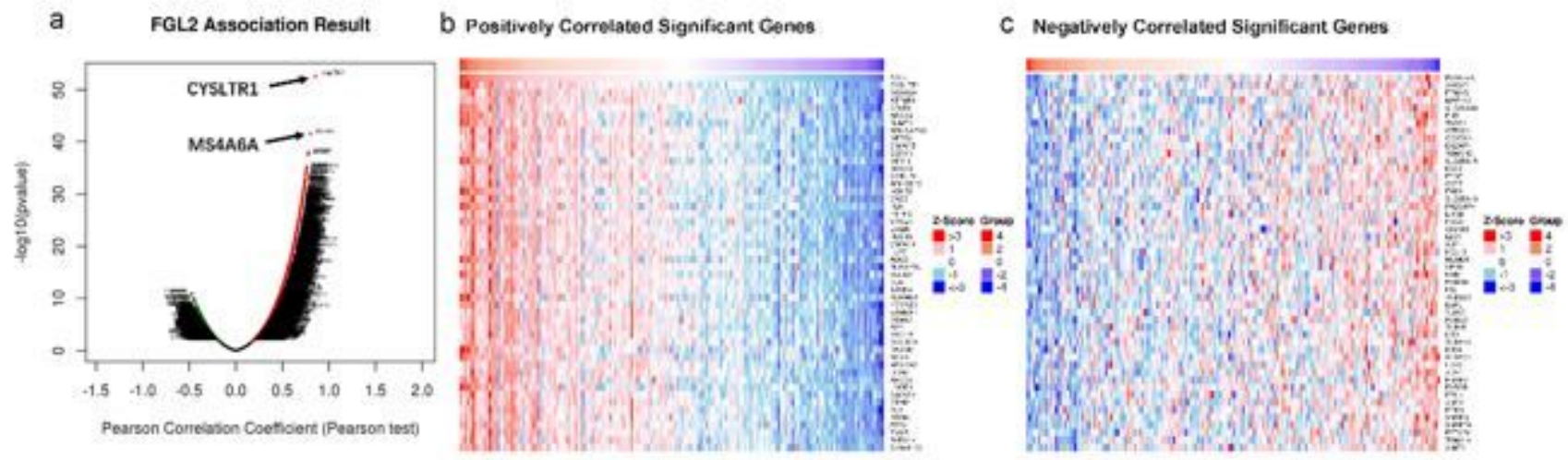

Figure 6 
Correlation between genes differentially expressed in ESCA with FGL2 expression using LinkedOmics. a. A Pearson test was used to analyse correlations between FGL2 and genes differentially expressed in ESCA. b-c. Heatmaps showing the top 50 genes that correlated positively and negatively with FGL2 in ESCA. Positively correlated genes are indicated in red, while negatively correlated genes are shown in blue.

a

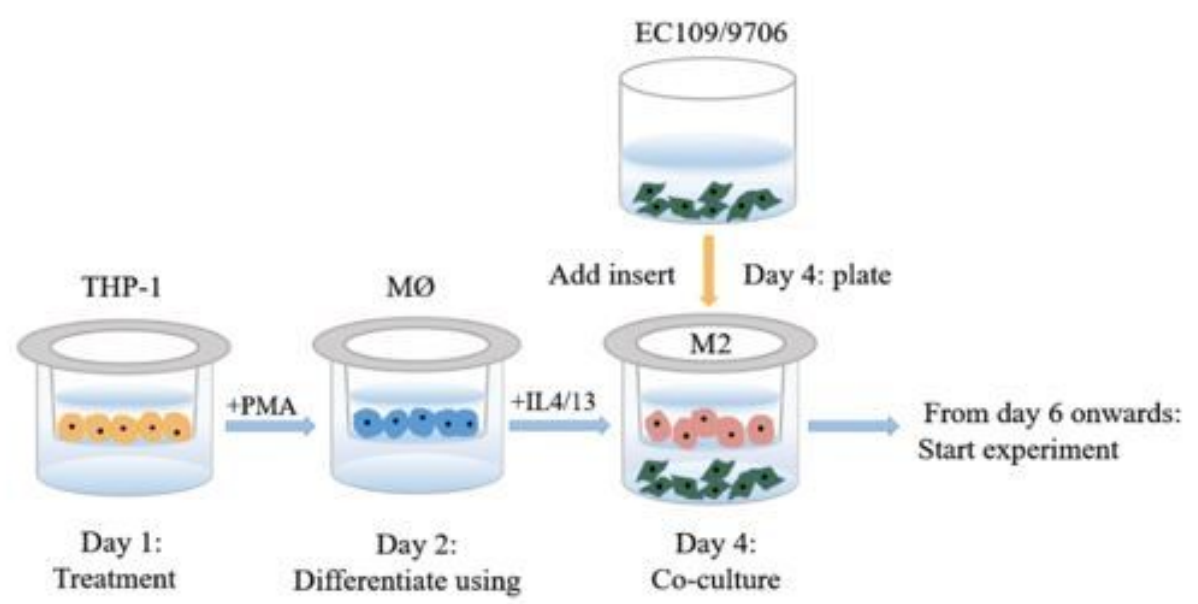

b

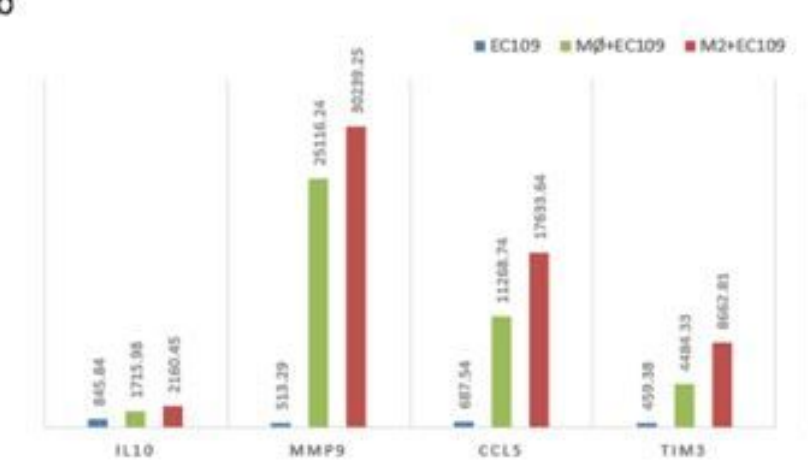

c

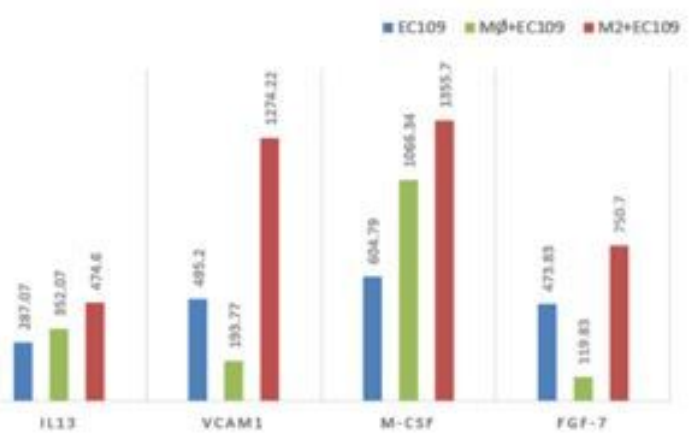

d

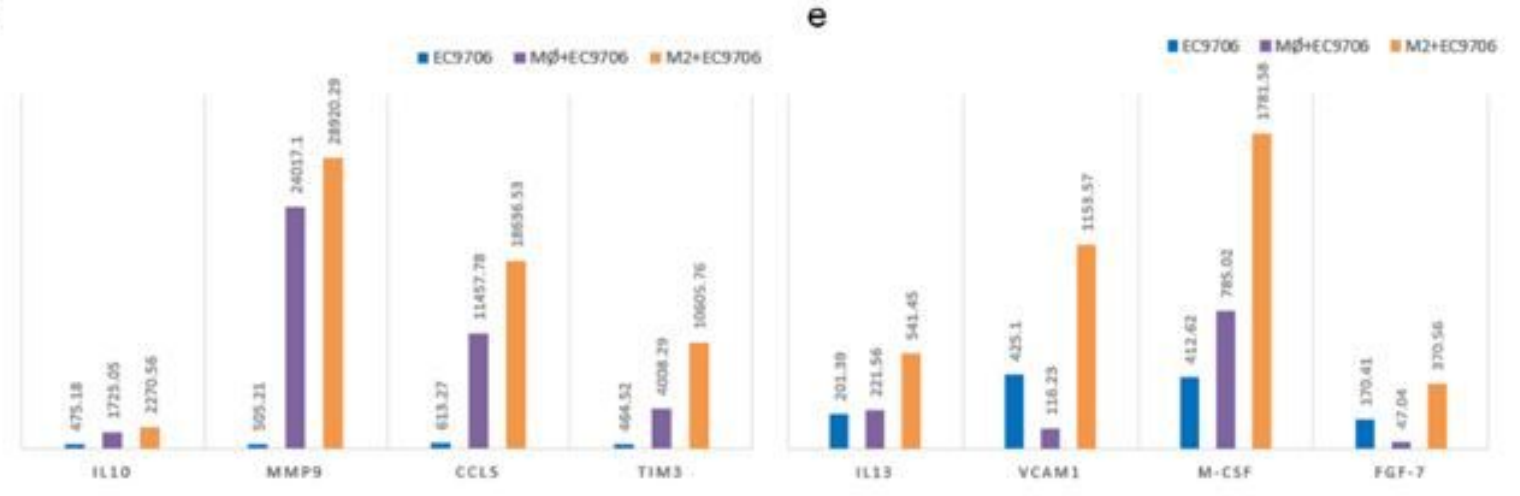

Figure 7

Identification of cytokines with significantly different expression levels in in vitro cell co-culture. a. Schematic of the process of co-culturing macrophages and ESCC cells (EC109 and EC9706). b-C. Expression level of IL-10, MMP9, CCL5, TIM3, IL-13, VCAM1, M-CSF and FGF-7 in the EC109 culture alone, co-culture of macrophages and EC109 cells, and co-culture of M2 macrophages and EC109 cells $₫ \mathrm{~d}-\mathrm{e}$. Expression levels of IL-10, MMP9, CCL5, TIM3, IL-13, VCAM1, M-CSF and FGF-7 in EC9706 culture alone, 


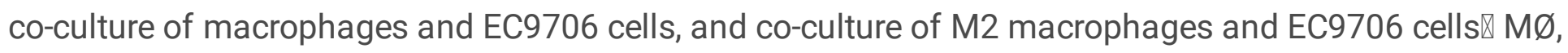
macrophage; M2, M2-like TAM
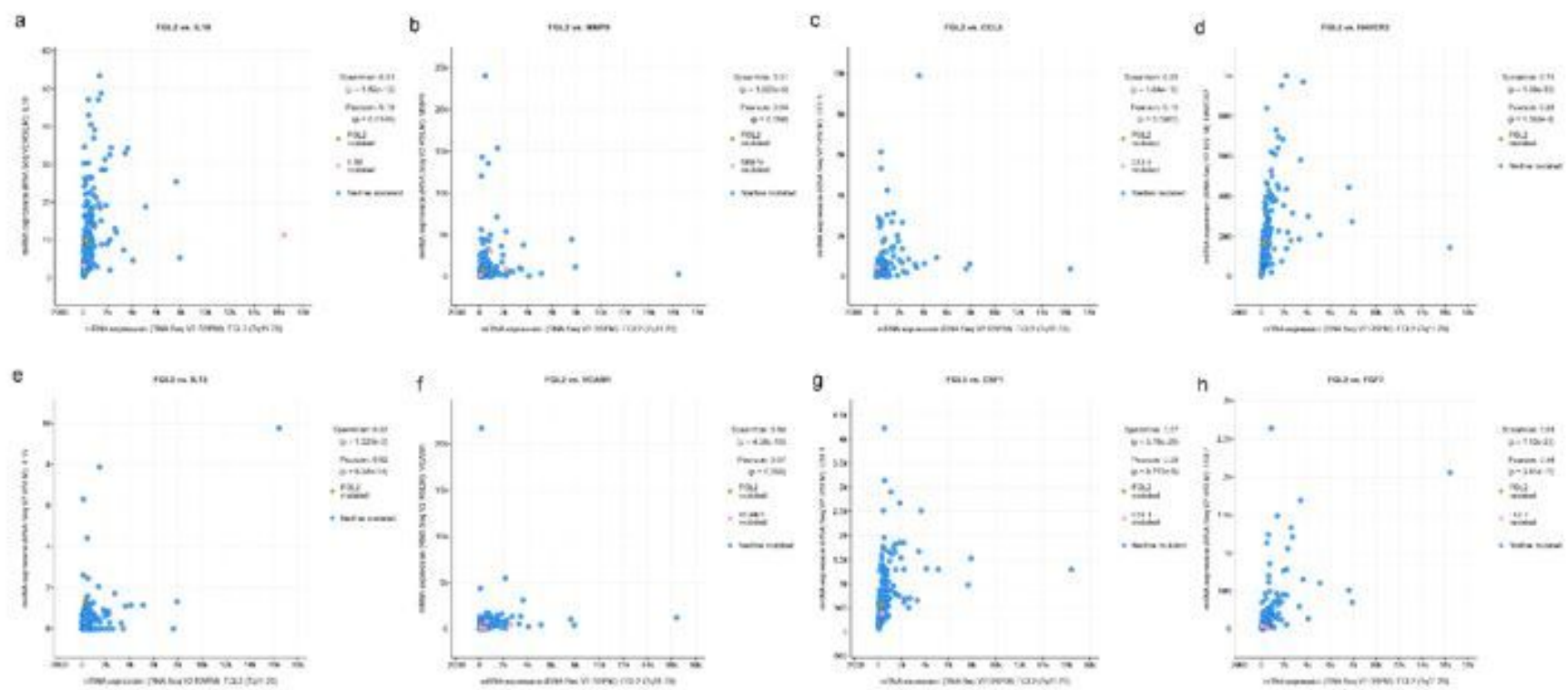

\section{Figure 8}

Correlation analysis between cytokines and FGL2 expression in ESCA using cBioPortal. a-h. Scatterplots of correlations between FGL2 expression and IL-10 (a), MMP9 (b), CCL5(c), TIM3(d), IL-13 (e), VCAM1 (f), M-CSF(CSF1) (g) and FGF-7 (h) expression. Spearman $\mathrm{P}<0.05$ was considered statistically significant. 
a

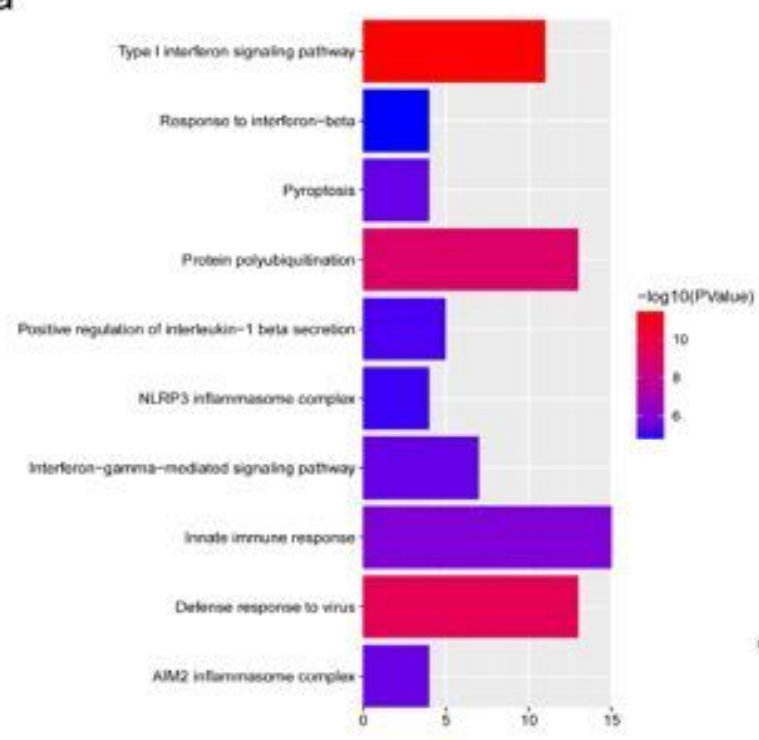

C

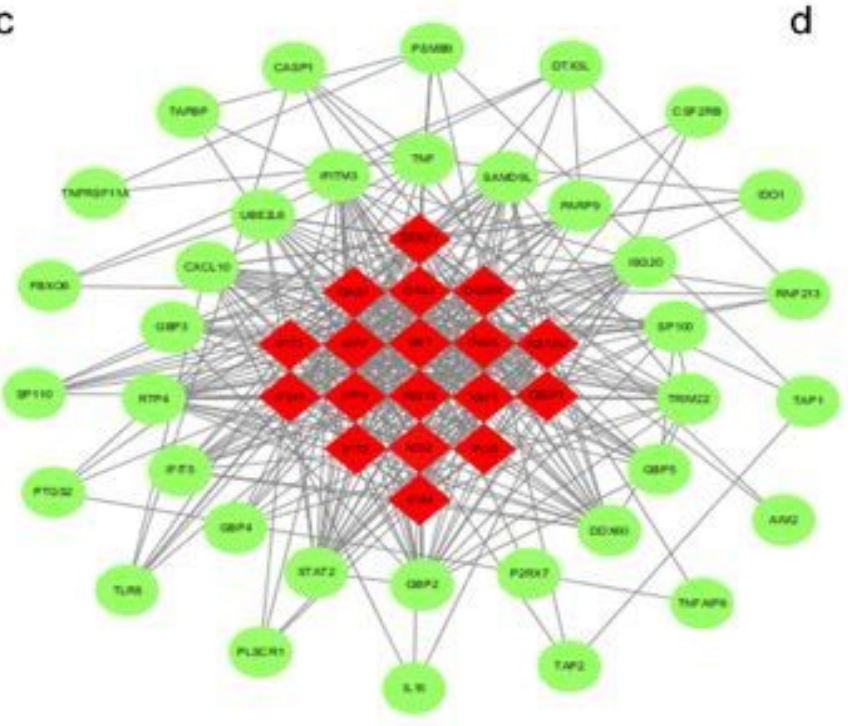

b

d
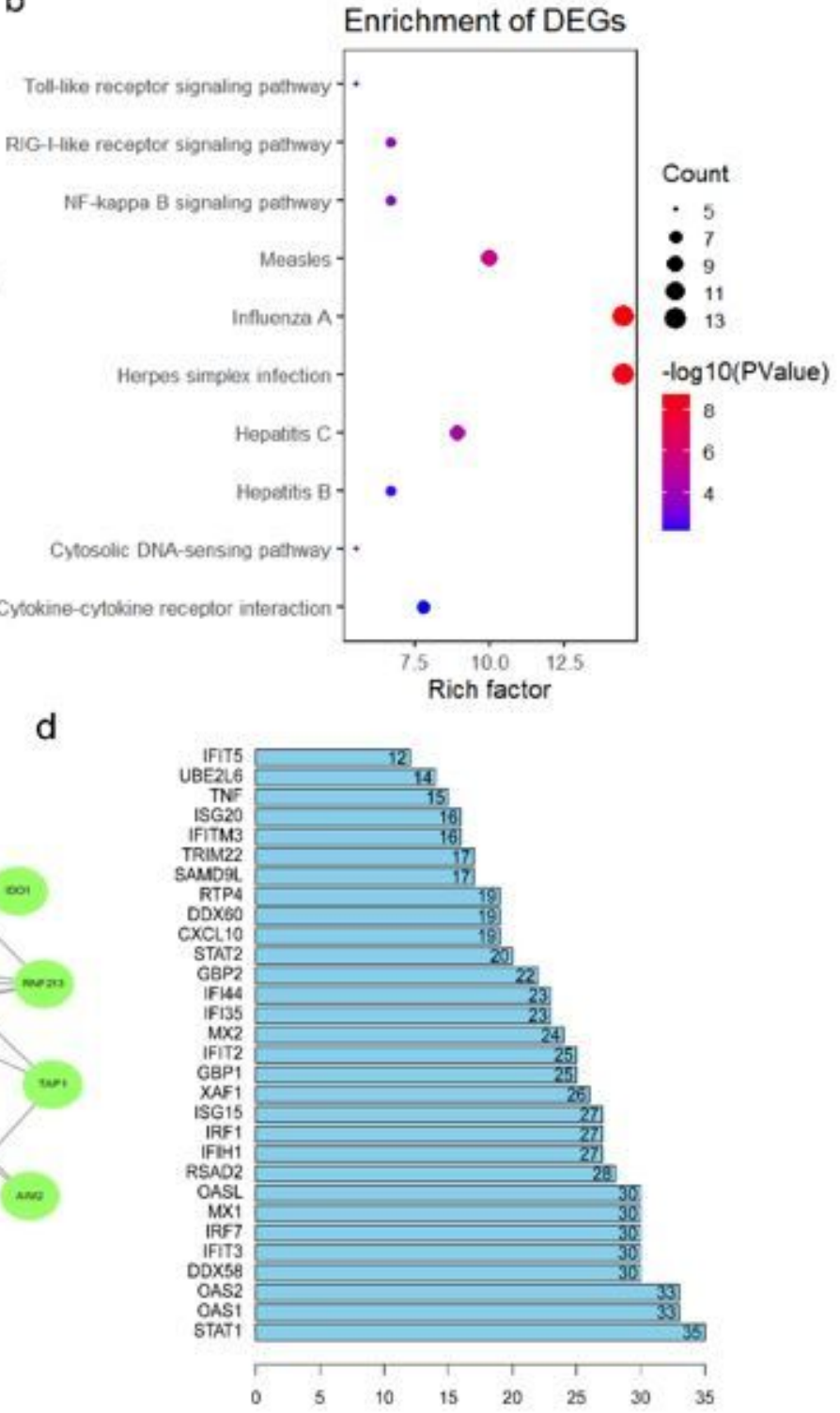

Figure 9

Analysis of DEGs in M1 and M2 macrophages. a-b. Histogram of top 10 GO terms (a) and bubble plot of top 10 KEGG pathways (b) enriched. The rank criterion had an FDR $<0.05$. The abscissa represents the percentage of DEGs enriched in each term or pathway, while the size represents the number of DEGs enriched in each item or pathway, and the colour represents the adjusted p-value. c. PPI network constructed 54 hub genes, with the red region representing the most closely connected module. A comprehensive Gt score $>0.7$ was considered statistically significant. $d$. Top 30 genes with greatest degree of differential expression. The ordinate lists the gene name and the abscissa represents the degree of differential expression. 


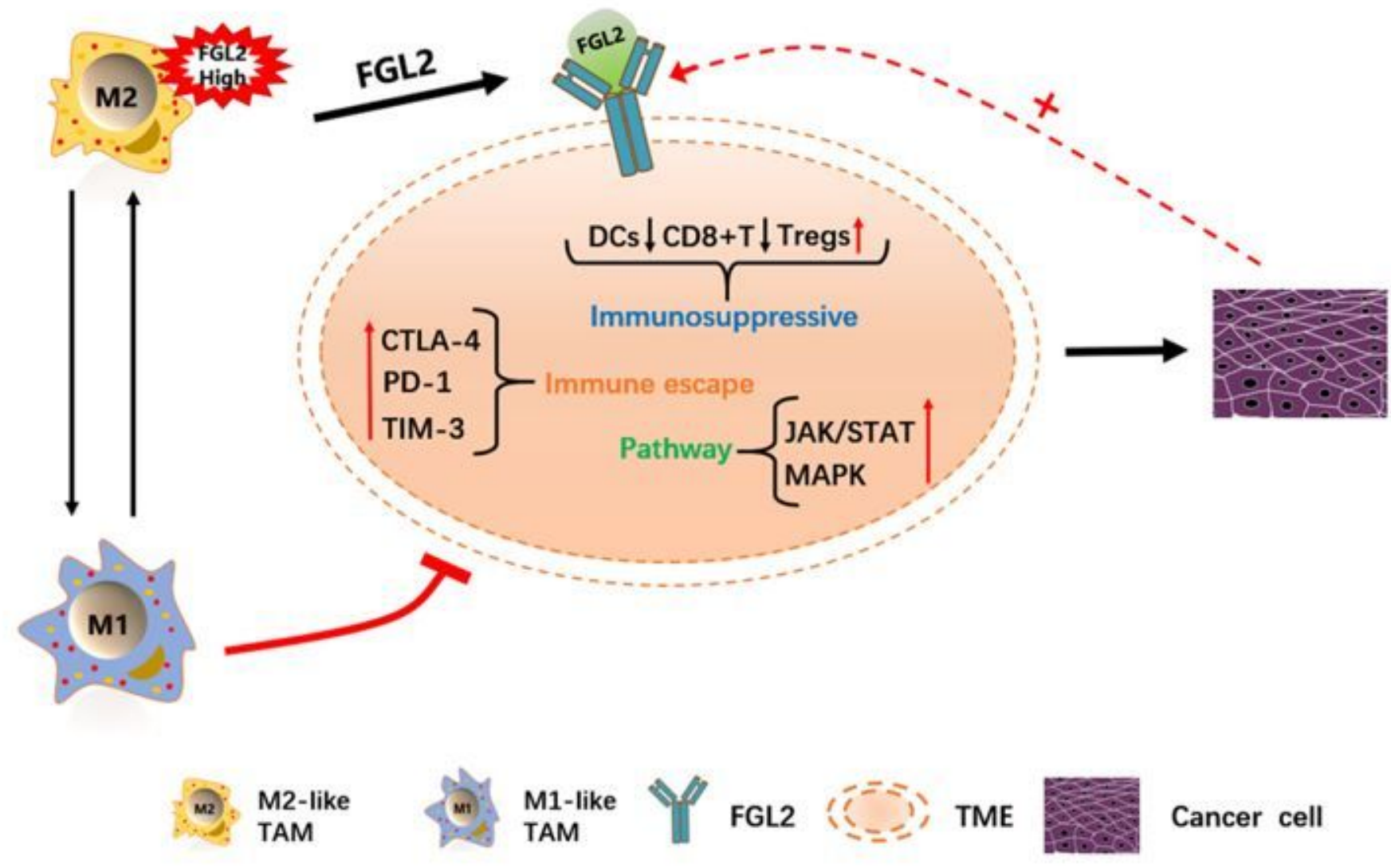

Figure 10

Schematic diagram depicting the potential role of FGL2 in esophageal carcinoma. M2-like TAMs recruit immune infiltrating cells by increasing FGL2 expression, producing a microenvironment that is conducive to tumour growth, and thus inducing the occurrence and development of ESCA. FGL2 expression is specifically correlated with immune cell infiltration and plays a vital role in immune escape in the esophageal cancer microenvironment. Reversing the polarization of tumour-associated macrophages into M1-like TAMs provides new targets for the development of tumour immunotherapies for treating esophageal carcinoma.

\section{Supplementary Files}

This is a list of supplementary files associated with this preprint. Click to download.

- Supplementaryinformation.docx 